Pacific

Journal of

Mathematics

NONCONCORDANT LINKS WITH HOMOLOGY COBORDANT ZERO-FRAMED SURGERY MANIFOLDS

Jae Choon Cha AND Mark Powell 


\title{
NONCONCORDANT LINKS WITH HOMOLOGY COBORDANT ZERO-FRAMED SURGERY MANIFOLDS
}

\author{
Jae Choon Cha ANd Mark Powell
}

\begin{abstract}
We use topological surgery theory to give sufficient conditions for the zeroframed surgery manifold of a 3-component link to be homology cobordant to the zero-framed surgery on the Borromean rings (also known as the 3torus) via a topological homology cobordism preserving the free homotopy classes of the meridians.

This enables us to give examples of 3-component links with unknotted components and vanishing pairwise linking numbers, such that any two of these links have homology cobordant zero-surgeries in the above sense, but the zero-surgery manifolds are not homeomorphic. Moreover, the links are not concordant to one another, and in fact they can be chosen to be height $h$ but not height $h+1$ symmetric grope concordant, for each $h$ which is at least three.
\end{abstract}

\section{Introduction}

It is well known that the study of homology cobordism of 3-manifolds is essential for understanding the concordance of knots and links: homology cobordism of the exteriors of links in $S^{3}$ is equivalent to concordance in a homology $S^{3} \times I$, and an additional mild normal generation condition for $\pi_{1}$ is equivalent to topological concordance in $S^{3} \times I$ (this also holds modulo the 4-dimensional Poincaré conjecture in the smooth case).

We recall the definitions: two $m$-component links $L_{0}$ and $L_{1}$ in $S^{3}$ are said to be topologically (respectively smoothly) concordant if there exist $m$ locally flat (respectively smoothly embedded) disjoint annuli in $S^{3} \times[0,1]$ cobounded by components of $L_{0} \times\{0\}$ and $-L_{1} \times\{1\}$. Two 3-manifolds $M_{0}$ and $M_{1}$ bordered by a 2-manifold $\Sigma$, that is, endowed with a marking $\mu_{i}: \Sigma \stackrel{\simeq}{\rightarrow} \partial M_{i}$, are topologically (respectively smoothly) homology cobordant if there is a topological (respectively smooth) 4-manifold $W$ with

$$
\partial W=M_{0} \sqcup-M_{1} \sqcup \Sigma \times[0,1] /\left(\mu_{0}(x) \sim x \times\{0\}, \mu_{1}(x) \sim x \times\{1\}, x \in \Sigma\right),
$$

MSC2010: 57M25, 57N70.

Keywords: homology cobordism, zero-framed surgery, topological surgery, link concordance, symmetric grope concordance. 
such that the inclusions $M_{i} \rightarrow W(i=0,1)$ induce isomorphisms on integral homology groups. In this paper links are oriented, and link exteriors are always bordered by $\bigsqcup_{m} S^{1} \times S^{1}$ under the zero framing.

In high dimensions, concordance classification results were obtained by studying homology surgery, with the aim of surgeries being to produce a homology cobordism of the exteriors (for example, see [Cappell and Shaneson 1974; 1980; Le Dimet 1988]). On the other hand, for knots and links in dimension three, the zero-surgery manifolds and their 4-dimensional homology cobordisms have been extensively used in the literature in order to understand the structure peculiar to low dimensions, especially in the topological category. Recall that performing zero-framed surgery on a link in $S^{3}$ yields a closed 3-manifold, called the zero-surgery manifold.

The classical invariants such as the knot signature and Levine's algebraic knot concordance class [Levine 1969a; 1969b] are obtained from the zero-surgery manifold of a knot, via the Blanchfield form. Also, higher-order knot concordance obstructions, such as Casson-Gordon invariants [Casson and Gordon 1978; 1986], and Cochran-Orr-Teichner $L^{2}$-signatures [Cochran et al. 2003] are obtained from the zero-surgery manifold (often together with the homology class of the meridian).

A natural interesting question is whether the homology cobordism class of a zero-surgery manifold determines the concordance class of a knot or link or if it determines the homology cobordism class of the exterior.

In this paper we show, in a strong sense involving homotopy of meridians, that the answer is negative for a large class of links satisfying a certain nonvanishing condition on Milnor's $\bar{\mu}$-invariants, even in the framework of symmetric grope and Whitney tower generalisations of concordance and homology cobordism in the sense of [Cochran et al. 2003; Cha 2014]. Also, we employ topological surgery in dimension 4 to give a new construction of homology cobordisms of zero-surgery manifolds. Next we state our main theorems, after which we will discuss these aspects further.

Theorem 1.1. Suppose $h \geq 3$. Then there are infinitely many 3-component links $L_{0}, L_{1}, \ldots$, with vanishing pairwise linking numbers and with unknotted components, satisfying the following for any $i \neq j$ :

(1) The zero-surgery manifolds $M_{L_{i}}$ and $M_{L_{j}}$ are not homeomorphic.

(2) There is a topological homology cobordism between $M_{L_{i}}$ and $M_{L_{j}}$ in which the $k$-th meridians of $L_{i}$ and $L_{j}$ are freely homotopic for each $k=1,2,3$.

(3) The links $L_{i}$ and $L_{j}$ are height $h$ but not height $h+1$ symmetric grope concordant. In particular, $L_{i}$ and $L_{j}$ are not concordant.

For a definition of height $h$ symmetric grope concordance, see Definition 4.2. Our links are obtained from the Borromean rings by performing a satellite construction 
along a curve lying in the kernel of the map $\pi_{1}\left(S^{3} \backslash L\right) \rightarrow \pi_{1}\left(M_{L}\right)$ induced by inclusion.

As a counterpoint to Theorem 1.1, we show that there are infinite families of links with the same nonvanishing Milnor invariants, with homeomorphic zerosurgery manifolds preserving the homotopy classes of the meridians, but which are not concordant.

The Milnor invariant of an $m$-component link associated to a multi-index $I=$ $i_{1} i_{2} \cdots i_{r}$ with $i_{j} \in\{1, \ldots, m\}$, as defined in [Milnor 1957], will be denoted by $\bar{\mu}_{L}(I)$. We denote its length by $|I|:=r$. Define $k(m):=\left\lfloor\log _{2}(m-1)\right\rfloor$.

Theorem 1.2. Let I be a multi-index with nonrepeating indices with length $m \geq 2$. For any $h \geq k(m)+2$ there are infinitely many m-component links $L_{0}, L_{1}, \ldots$, with unknotted components, satisfying the following:

(1) The $L_{i}$ have identical $\bar{\mu}$-invariants, $\bar{\mu}_{L_{i}}(I)=1$, and $\bar{\mu}_{L_{i}}(J)=0$ for $|J|<|I|$.

(2) There is a homeomorphism between the zero-surgery manifolds $M_{L_{i}}$ and $M_{L_{j}}$ which preserves the homotopy classes of the meridians.

(3) The links $L_{i}$ and $L_{j}$ are height $h$ but not height $h+1$ symmetric grope concordant. In particular, $L_{i}$ and $L_{j}$ are not concordant.

The case when $m \geq 3$ should be compared with Theorem 1.1 since then the links $L_{i}$ have vanishing pairwise linking numbers. To construct such links we start with certain iterated Bing doubles constructed using T. Cochran's algorithm, which realise the Milnor invariant required. We then perform satellite operations which affect the concordance class of the link but do not change the homeomorphism type of the zero-surgery manifold.

We remark that we could also phrase Theorems 1.1 and 1.2 in terms of symmetric Whitney tower concordance instead of grope concordance.

In the three subsections below, we discuss some features of Theorem 1.1, regarding (i) the use of topological surgery in dimension 4, (ii) link concordance versus zero-surgery homology cobordism, and (iii) link exteriors and the homology surgery approach.

1A. Topological surgery for 4-dimensional homology cobordism. An interesting aspect of the proof of Theorem 1.1 is that we employ topological surgery in dimension 4 to give a sufficient condition for certain zero-surgery manifolds of 3-component links to be homology cobordant. It is well known that topological surgery in dimension 4 is useful for obtaining homology cobordisms (and consequently concordances), although the current state of the art in terms of "good" groups, for which the $\pi_{1}$-null disc lemma is known, is still insufficient for the general case. M. Freedman showed that knots of Alexander polynomial one are concordant to the unknot [Freedman and Quinn 1990, Theorem 11.7B]. J. Davis 
[2006] extended the program to show that 2-component links with Alexander polynomial one are concordant to the Hopf link. These two cases use topological surgery over fundamental groups $\mathbb{Z}$ and $\mathbb{Z}^{2}$, respectively. Due to the rarity of good groups for 4-dimensional topological surgery, there are not many other situations where such positive results on knot and link concordance can currently be proven. As another case, S. Friedl and P. Teichner [2005] found sufficient conditions for a knot to be homotopy ribbon, and in particular slice, with a certain ribbon group $\mathbb{Z} \ltimes \mathbb{Z}\left[\frac{1}{2}\right]$.

We give another instance of the utility of topological surgery for constructing homology cobordisms, using the group $\mathbb{Z}^{3}$, which is manageable from the point of view of topological surgery in dimension 4. Indeed, our sufficient condition for zero-surgery manifolds to be homology cobordant focuses on the Borromean rings as a base link. The zero-surgery manifold $M_{\mathrm{Bor}}$ of the Borromean rings is the 3-torus $T^{3}=S^{1} \times S^{1} \times S^{1}$, whose fundamental group is $\mathbb{Z}^{3}$.

To state our result, we with the following notation: Let

$$
\Lambda:=\mathbb{Z}\left[\mathbb{Z}^{3}\right]=\mathbb{Z}\left[t_{1}^{ \pm 1}, t_{2}^{ \pm 1}, t_{3}^{ \pm 1}\right] .
$$

Denote the zero-surgery manifold of a link $L$ by $M_{L}$ as before. For a 3-component link $L$ with vanishing pairwise linking numbers, there is a canonical homotopy class of maps $f_{L}: M_{L} \rightarrow M_{\mathrm{Bor}}=T^{3}$ which send the homotopy class of the $i$-th meridian of $L$ to that of the Borromean rings, namely the $i$-th circle factor of $T^{3}$. After choosing an identification of $\pi_{1}\left(T^{3}\right)=\mathbb{Z}^{3}$, we can use this to define the $\Lambda$ coefficient homology $H_{1}\left(M_{L} ; \Lambda\right)$. We say that a map $f: M_{L} \rightarrow T^{3}$ is a $\Lambda$-homology equivalence if $f$ is homotopic to $f_{L}$ and $f$ induces isomorphisms on $H_{*}(-; \Lambda)$.

Theorem 1.3. Suppose $L$ is a 3-component link whose components have trivial Arf invariants and there exists a $\Lambda$-homology equivalence $M_{L} \rightarrow T^{3}$. Then there is a homology cobordism $W$ between $M_{L}$ and $T^{3}=M_{\text {Bor }}$ for which the inclusioninduced maps $\pi_{1}\left(M_{L}\right) \rightarrow \pi_{1}(W) \stackrel{\simeq}{\leftarrow} \pi_{1}\left(T^{3}\right)$ are such that the composition from left to right takes meridians to meridians.

1B. Link concordance versus zero-surgery homology cobordism. We review the general question of whether links with homology cobordant zero-surgery manifolds are concordant. The answer to the basic question is easily seen to be no, once one knows of a result of C. Livingston [1983] that there are knots not concordant to their reverses. Note that a knot and its reverse have the same zero-surgery manifold. This leads us to consider some additional conditions on the homology cobordism, involving the meridians. In what follows, meridians are always positively oriented.

First, observe that the exteriors of two links are homology cobordant if and only if the zero-framed meridians cobound framed annuli disjointly embedded in a homology cobordism of the zero-surgery manifolds. (For the if direction, note that the exterior of the framed annuli is a homology cobordism of the link exteriors.) 
In particular, it holds if two links (or knots) are concordant.

Regarding the knot case, in [Cochran et al. 2013], T. Cochran, B. Franklin, M. Hedden and P. Horn considered homology cobordisms of zero-surgery manifolds in which the meridians are homologous: in the smooth category, they showed that the existence of such a homology cobordism is insufficient for knots to be concordant. In the topological case this is still left unknown.

Concerning a stronger homotopy analogue, the following is unknown in both the smooth and topological cases:

Question 1.4. If there is a homology cobordism of zero-surgery manifolds of two knots in which the meridians are homotopic, are the knots concordant? Or concordant in a homology $S^{3} \times I$ ?

For the link case, results in the literature give nonconcordant examples whose zero-surgery manifolds admit a homology cobordism with homotopic meridians. As a generic example in the topological category, consider a 2-component link with linking number one. The zero-surgery manifold is a homology 3-sphere, which bounds a contractible topological 4-manifold by [Freedman and Quinn 1990, Corollary 9.3C]. Taking the connected sum of such 4-manifolds, one obtains the following: the zero-surgery manifolds of any two linking number one 2-component links cobound a simply connected topological homology cobordism. Note that in this case the meridians are automatically homotopic. There are many linking number one 2-component links which are not concordant, as can be detected, for example, by the multivariable Alexander polynomial [Kawauchi 1978; Nakagawa 1978]. For related in-depth study, the reader is referred to, for instance, [Cha and Ko 1999; Friedl and Powell 2011; Cha 2014]. With our respective coauthors, we gave nonconcordant linking number one links with two unknotted components, for which abelian invariants such as the multivariable Alexander polynomial are unable to obstruct them from being concordant.

There are other examples which have knotted components: in [Cochran et al. 2013, end of Section 1], the authors discuss 2-component linking number zero links with homeomorphic zero-surgery manifolds which have nonconcordant (knotted) components. These links are obviously not concordant, and it can be seen that the homeomorphisms preserve meridians up to homotopy.

By contrast with the above examples, our links have unknotted components and vanishing pairwise linking numbers. Another feature exhibited by the links of Theorems 1.1 and 1.2 is that the entire subtlety of symmetric grope concordance of links can occur, within a single homology cobordism/homeomorphism class of the zero-surgeries, even modulo local knot tying.

We remark that all the links of Theorems 1.1 and 1.2 lie in the same " $k$-solvequivalence class" for all $k$ in the sense of [Cochran and Kim 2008, Definition 2.5]. 
1C. Link exteriors and the homology surgery approach. Our results serve to underline the philosophy that when investigating the relative problem of whether two links are concordant, and neither of them are the unlink, one should consider obstructions to homology cobordism of the link exteriors viewed as bordered manifolds, rather than to homology cobordism of the zero-surgery manifolds, even in low dimensions. This was implemented in, for example, [Kawauchi 1978; Nakagawa 1978; Cha 2014] (see also [Friedl and Powell 2011] for a related approach).

Although we stated our results in terms of grope concordance of links in Theorems 1.1 and 1.2 given above, in fact we show more: the link exteriors are far from being homology cobordant, as measured in terms of Whitney towers. A more detailed discussion is given in Section 5. For the purpose of distinguishing exteriors, we use the amenable Cheeger-Gromov $\rho$-invariant technology for bordered 3manifolds (particularly for link exteriors) developed in [Cha 2014], generalising applications of $\rho$-invariants to concordance and homology cobordism in [Cochran et al. 2003; 2009; Cha and Orr 2012].

We will now discuss our results from the viewpoint of the homology surgery approach to link concordance classification, initiated by S. Cappell and J. Shaneson [1974; 1980] and implemented in high dimensions by J. Le Dimet [1988] using P. Vogel's homology localisation of spaces [1978]. The strategy consists of two parts. Consider the problem of comparing two given link exteriors. First we decide whether the exteriors have the same "Poincaré type", which roughly means that they have homotopy-equivalent Vogel homology localisations. If so, there is a common finite target space, into which the exteriors are mapped by homology equivalences rel. boundary. Once this is the case, a surgery problem is defined, and one can try to decide whether homology surgery gives a homology cobordism of the exteriors. The first step is obstructed by homotopy invariants (including Milnor $\bar{\mu}$-invariants in low dimension). The failure of the second step is measured by surgery obstructions, which are not yet fully formulated in low dimension (even modulo the fact that 4-dimensional surgery might not work), since the fundamental group plays a more sophisticated central rôle; see [Powell 2012] for the beginning of an algebraic surgery approach to this problem in the context of knot slicing.

Our examples illustrate that for many Poincaré types, namely those in Theorems 1.1 and 1.2, we get a rich theory of surgery obstructions within each Poincaré type, which is invisible via zero-surgery manifolds. We remark that for our links $L_{i}$ in Theorems 1.1 and 1.2, there is a homology equivalence of the exterior of each $L_{i}$ into that of a fixed one, say $L_{1}$, since we use satellite constructions (see Section 4). It follows that the exteriors have the same Poincaré type in the above sense. In this paper, (parts of the not yet fully formulated) homology surgery obstructions in dimension 4 have their incarnation in Theorem 5.2, the Amenable Signature Theorem. 
Organisation of the paper. In Section 2, we explore the implications of the hypothesis that a homology equivalence $f: M_{L} \rightarrow T^{3}$ as in Theorem 1.3 exists, and we prove Theorem 1.3 in Section 3. In Section 4, we construct links with a given Milnor invariant with nonrepeating indices, and perform satellite operations on the links to construct the links of Theorems 1.1 and 1.2, which are height $h$ symmetric grope concordant. In Section 5, we show that none of these links are height $h+1$ grope concordant to one another.

\section{Homology type of zero-surgery manifolds and the 3-torus}

This section discusses the hypotheses of Theorem 1.3. We begin the section by briefly reminding the reader who is familiar with Kirby calculus of a nice way to see the following fact.

Lemma 2.1. The zero-surgery manifold of the Borromean rings is homeomorphic to the 3-torus.

Proof. Place dots on two components of the Borromean rings and a zero near the other. Each component of the Borromean rings is a commutator in the meridians of the other two components, so this is a Kirby diagram for $T^{2} \times D^{2}$, whose boundary is $T^{3}$. The 1-handles (dotted circles) can be replaced with zero-framed 2-handles without changing the boundary.

In the following proposition we expand on the meaning and implications of the condition in Theorem 1.3. Denote the exterior of a link $L$ by $X_{L}:=S^{3} \backslash \nu L$ as before.

Proposition 2.2. Suppose that $L$ is a 3-component link. Then the following are equivalent:

(1) There is a $\Lambda$-homology equivalence $f: M_{L} \rightarrow T^{3}$.

(2) The preferred longitudes generate the link module $H_{1}\left(X_{L} ; \Lambda\right)$.

(3) The pairwise linking numbers of $L$ vanish and $H_{1}\left(M_{L} ; \Lambda\right)=0$.

Furthermore, (any of) the above conditions imply that L has multivariable Alexander polynomial $\Delta_{L}=\left(t_{1}-1\right)\left(t_{2}-1\right)\left(t_{3}-1\right)$, and this implies that the Milnor invariant $\bar{\mu}_{L}(123)$ is equal to \pm 1 .

Proof. First we will observe that (2) and (3) are equivalent. Longitudes of $L$ represent elements in $H_{1}\left(X_{L} ; \Lambda\right) \cong \pi_{1}\left(X_{L}\right)^{(1)} / \pi_{1}\left(X_{L}\right)^{(2)}$ if and only if they are zero in $H_{1}\left(X_{L} ; \mathbb{Z}\right) \cong \mathbb{Z}^{3}$; that is, the pairwise linking numbers are zero. If this is the case, $H_{1}\left(M_{L} ; \Lambda\right)$ is isomorphic to $H_{1}\left(X_{L} ; \Lambda\right) /\langle$ longitudes $\rangle$, since $M_{L}$ is obtained by attaching three 2-handles to $E_{L}$ along the longitudes and then attaching three 3-handles along the boundary. It follows that longitudes generate $H_{1}\left(X_{L} ; \Lambda\right)$ if and only if $H_{1}\left(M_{L} ; \Lambda\right)=0$. 
Suppose (1) holds. Denote the meridians of $L$ by $\mu_{i}(i=1,2,3)$ and the linking number of the $i$-th and $j$-th components by $\ell_{i j}$. The $i$-th longitude $\lambda_{i}$, which is homologous to $\sum_{j \neq i} \ell_{i j} \mu_{i}$, is zero in $H_{1}\left(M_{L} ; \mathbb{Z}\right) \cong H_{1}\left(T^{3} ; \mathbb{Z}\right)$. Since $\left\{f_{*}\left(\left[\mu_{i}\right]\right)\right\}$ forms a basis of $H_{1}\left(T^{3} ; \mathbb{Z}\right) \cong \mathbb{Z}^{3}$, it follows by linear independence that $\ell_{i j}=0$ for any $i$ and $j$. Also, $H_{1}\left(M_{L} ; \Lambda\right) \cong H_{1}\left(T^{3} ; \Lambda\right)=0$. This shows that (3) holds.

Suppose (3) holds. Start with a map $g: \partial X_{L}=\bigsqcup_{3} S^{1} \times S^{1} \rightarrow T^{3}$ that sends $\mu_{i}$ to the $i$-th $S^{1}$ factor and $\lambda_{i}$ to a point. Observe that $g_{*}: H_{1}\left(\partial X_{L} ; \mathbb{Z}\right) \rightarrow H_{1}\left(T^{3} ; \mathbb{Z}\right)$ factors through the inclusion-induced map $i_{*}: H_{1}\left(\partial X_{L} ; \mathbb{Z}\right) \rightarrow H_{1}\left(M_{L} ; \mathbb{Z}\right)$ and the identifications $H_{1}\left(M_{L} ; \mathbb{Z}\right) \stackrel{\simeq}{\rightarrow} \mathbb{Z}^{3} \stackrel{\simeq}{\leftarrow} H_{1}\left(T^{3} ; \mathbb{Z}\right)$; this follows from the fact that $H_{1}\left(\partial X_{L} ; \mathbb{Z}\right) \cong \mathbb{Z}^{6}$ is generated by the $\mu_{i}$ and $\lambda_{i}$ and that both $g_{*}$ and $i_{*}$ are quotient maps, with their kernels generated by the $\lambda_{i}$. Since $T^{3}$ is a $K\left(\mathbb{Z}^{3}, 1\right)$, elementary obstruction theory shows that $g$ extends to a map $f: M_{L} \rightarrow T^{3}$.

Consider the universal coefficient spectral sequence (see, e.g., [Levine 1977, Theorem 2.3]) $E_{p, q}^{2}=\operatorname{Ext}_{\Lambda}^{p}\left(H_{q}\left(M_{L} ; \Lambda\right), \Lambda\right) \Rightarrow H^{n}\left(M_{L} ; \Lambda\right)$. We have $E_{0,1}^{2}=0$ since $H_{1}\left(M_{L} ; \Lambda\right)=0$, and $E_{1,0}^{2}=\operatorname{Ext}_{\Lambda}^{1}(\mathbb{Z}, \Lambda)=H^{1}\left(T^{3} ; \Lambda\right)=0$. It follows that $H^{1}\left(M_{L} ; \Lambda\right)=0$. By duality, $H_{2}\left(M_{L} ; \Lambda\right)=0$. Also, $H_{3}\left(M_{L} ; \Lambda\right)=0$ since the $\mathbb{Z}^{3}$-cover of $M_{L}$ is noncompact. Since $H_{0}\left(M_{L} ; \Lambda\right) \cong \mathbb{Z} \cong H_{0}\left(T^{3} ; \Lambda\right)$ and $H_{i}\left(T^{3} ; \Lambda\right)=0$ for $i>0$, it follows that $f$ is a $\Lambda$-homology equivalence. This completes the proof of the equivalence of (1), (2) and (3).

Suppose (1), (2) and (3) hold. Recall that the scalar multiplication of a loop by $t_{i}$ in the module $H_{1}\left(X_{L} ; \Lambda\right)$ is defined to be conjugation by $\mu_{i}$. Since $\lambda_{i}$ and $\mu_{i}$ commute, we have $\left(t_{i}-1\right) \lambda_{i}=0$ in $H_{1}\left(X_{L} ; \Lambda\right)$. From this and (2), it follows that there is an epimorphism of $A:=\bigoplus_{i=1}^{3} \Lambda /\left\langle t_{i}-1\right\rangle$ onto $H_{1}\left(X_{L} ; \Lambda\right)$. Since the zeroth elementary ideal of $A$ is the principal ideal generated by $\left(t_{1}-1\right)\left(t_{2}-1\right)\left(t_{3}-1\right)$, it follows that $\Delta_{L}$ is a factor of $\left(t_{1}-1\right)\left(t_{2}-1\right)\left(t_{3}-1\right)$. We now invoke the Torres condition (see, e.g., [Kawauchi 1996, Theorem 7.4.1]):

$$
\Delta_{L}\left(1, t_{2}, t_{3}\right)=\left(t_{2}^{\ell_{12}} t_{3}^{\ell_{13}}-1\right) \Delta_{L^{\prime}}\left(t_{2}, t_{3}\right),
$$

where $L^{\prime}$ is the sublink of $L$ with the first component deleted and $\ell_{i j}$ is the pairwise linking number. Since $\ell_{i j}=0$ by (3), we have $\Delta_{L}\left(1, t_{2}, t_{3}\right)=0$. It follows that $t_{1}-1$ is a factor of $\Delta_{L}$. Similarly $t_{2}-1$ and $t_{3}-1$ are factors. Therefore $\Delta_{L}\left(t_{1}, t_{2}, t_{3}\right)=\left(t_{1}-1\right)\left(t_{2}-1\right)\left(t_{3}-1\right)$.

To show the last part, suppose that $\Delta_{L}\left(t_{1}, t_{2}, t_{3}\right)=\left(t_{1}-1\right)\left(t_{2}-1\right)\left(t_{3}-1\right)$. By [Kawauchi 1996, Proposition 7.3.14], the single-variable Alexander polynomial $\Delta_{L}(t)$ of $L$ is given by

$$
\Delta_{L}(t)=(t-1) \Delta_{L}(t, t, t)=(t-1)^{4} \doteq\left((\sqrt{t})^{-1}-\sqrt{t}\right)^{4} .
$$

It follows that $L$ has Conway polynomial $\nabla_{L}(z)=z^{4}$, by the standard substitution $z=(\sqrt{t})^{-1}-\sqrt{t}$. Cochran [1985, Theorem 5.1] identified the coefficient of $z^{4}$ 
in $\nabla_{L}(z)$ with $\left(\mu_{L}(123)\right)^{2}$ for 3-component links with pairwise linking number zero. Applying this to our case, it follows that $\bar{\mu}_{L}(123)= \pm 1$.

\section{Construction of homology cobordisms using topological surgery}

This section gives the proof of Theorem 1.3. The proof will use surgery theory, and will parallel the proof given by Davis [2006] (see also [Hillman 2002, Section 7.6]). We will provide some details in order to fill in where the treatment in [Davis 2006] was terse, and to convince ourselves that the analogous arguments work in the case of interest.

For the convenience of the reader we restate Theorem 1.3 here.

Theorem 1.3. Suppose $L$ is a 3-component link whose components have trivial Arf invariants and there exists a $\Lambda$-homology equivalence $M_{L} \rightarrow T^{3}$. Then there is a homology cobordism $W$ between $M_{L}$ and $T^{3}=M_{\text {Bor }}$ for which the inclusioninduced maps $\pi_{1}\left(M_{L}\right) \rightarrow \pi_{1}(W) \stackrel{\simeq}{\leftarrow} \pi_{1}\left(T^{3}\right)$ are such that the composition from left to right takes meridians to meridians.

Remark 3.2. It is an interesting question to determine whether there are extra conditions which can be imposed in order to see that the Arf invariants of the components are forced to vanish by the homological assumptions. In the cases of knots and 2-component links with Alexander polynomial one, the Arf invariants of the components are automatically trivial. For knots, $\Delta_{K}(-1)$ computes the Arf invariant, by [Levine 1966]. For 2-component links one observes that $\Delta_{L}(t, 1)$ and $\Delta_{L}(1, t)$ give the Alexander polynomials of the components, by the Torres condition, and then applies Levine's theorem. These arguments do not seem to extend to the 3-component case of current interest.

The proof of Theorem 1.3 will occupy the rest of this section. In order to produce a homology cobordism, we will first show that there is a normal cobordism between normal maps $f: M_{L} \rightarrow T^{3}$ and Id $: T^{3} \rightarrow T^{3}$. Interestingly, we can work with smooth manifolds in order to establish the existence of a normal cobordism. This will make arguments which invoke tangent bundles and transversality easier to digest. Only at the end of the proof of Theorem 1.3, where we take connected sums with the $E_{8}$-manifold, and where we claim that the vanishing of a surgery obstruction implies that surgery can be done, do we need to leave the realm of smooth manifolds.

Definition 3.3. Let $X$ be an $n$-dimensional manifold with a vector bundle $v \rightarrow X$. A degree-one normal map $(F, b)$ over $X$ is an $n$-manifold $M$ with a map $F: M \rightarrow X$ which induces an isomorphism $F_{*}: H_{n}(M ; \mathbb{Z}) \stackrel{\simeq}{\rightarrow} H_{n}(X ; \mathbb{Z})$, together with a stable trivialisation $b: T M \oplus F^{*} v \oplus \varepsilon^{l} \cong \varepsilon^{k}$. 
A degree-one normal cobordism $(J, e)$ between normal maps $(F: M \rightarrow X, b)$ and $(G: N \rightarrow X, c)$ is an $(n+1)$-dimensional cobordism between $M$ and $N$ with a map $J: W \rightarrow X \times I$ extending $F: M \rightarrow X \times\{0\}$ and $G: M \rightarrow X \times\{1\}$, which induces an isomorphism

$$
J_{*}: H_{n+1}(W, \partial W ; \mathbb{Z}) \stackrel{\simeq}{\longrightarrow} H_{n+1}(X \times I, X \times\{0,1\} ; \mathbb{Z}),
$$

together with a stable trivialisation $e: T W \oplus J^{*}(\nu \times I) \oplus \varepsilon^{l^{\prime}} \cong \varepsilon^{k^{\prime}}$.

For us, let $X=T^{3}$, and let $v$ be its tangent bundle. We fix a framing on the stable tangent bundle of the target torus $T^{3}$ once and for all. Note that this canonically determines a trivialisation of the tangent bundle of $F^{*} \nu$, for any map $F: M \rightarrow X$, by the following diagram, in which the bottom composition is the constant map, denoted $*$, and the top composition is the pull back $F^{*} v$. The middle composition is the induced framing.

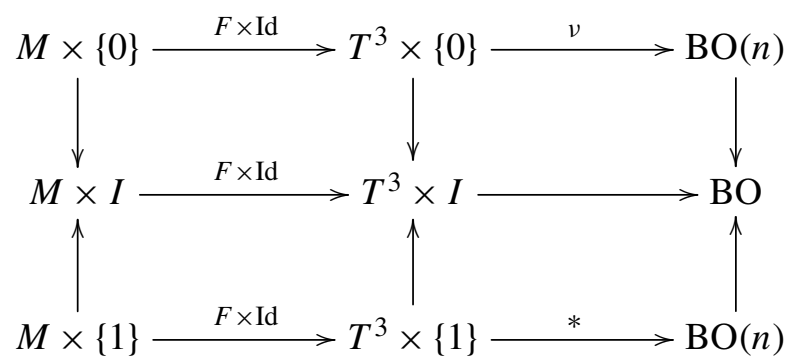

A framing of the tangent bundle of the domain therefore determines a normal map.

Lemma 3.4. Let $L$ be a link whose components have trivial Arf invariants, and let $f: M_{L} \rightarrow T^{3}$ be a degree-one normal map which induces a $\mathbb{Z}$-homology isomorphism and which maps a chosen meridian $\mu_{i}$ to the $i$-th $S^{1}$ factor of $T^{3}$ for $i=1,2,3$. We can make a homotopy of $f$ and choose a framing on $M_{L}$ so that $f: M_{L} \rightarrow T^{3}$ and $\mathrm{Id}: T^{3} \rightarrow T^{3}$ are degree-one normal cobordant.

Proof. We need to show that we can choose a framing on $M_{L}$ such that the disjoint union $M_{L} \sqcup-T^{3}$ represent the trivial element of $\Omega_{3}^{\mathrm{fr}}\left(T^{3}\right)$. We compute this bordism group:

$$
\widetilde{\Omega}_{3}^{\mathrm{fr}}\left(T^{3}\right) \cong \widetilde{\Omega}_{4}^{\mathrm{fr}}\left(\Sigma T^{3}\right) \cong \widetilde{\Omega}_{4}^{\mathrm{fr}}\left(S^{2} \vee S^{2} \vee S^{2} \vee S^{3} \vee S^{3} \vee S^{3} \vee S^{4}\right),
$$

with this last isomorphism induced by a homotopy equivalence of spaces. There is a copy of $S^{i+1}$ for each $i$-cell of $T^{3}$, for $i=1,2,3$. To see this homotopy equivalence, we need to see that the attaching maps of the cells are null-homotopic. The suspension of the 1-skeleton of $T^{3}$ is $S^{2} \vee S^{2} \vee S^{2}$. The Hilton-Milnor theorem [Hilton 1955, Theorem A] computes the homotopy groups of a wedge of spheres. 
The attaching maps for the 2-cells of $T^{3}$ become the attaching maps for the 3-cells of $\Sigma T^{3}$, namely maps in

$$
\pi_{2}\left(S^{2} \vee S^{2} \vee S^{2}\right) \cong \bigoplus_{3} \pi_{2}\left(S^{2}\right) \cong \bigoplus_{3} \mathbb{Z},
$$

where the first isomorphism is by the Hilton-Milnor theorem. The commutator attaching maps become trivial in the abelian $\pi_{2}\left(S^{2}\right)$. Therefore the 3-skeleton of $\Sigma T^{3}$ is a wedge $S^{2} \vee S^{2} \vee S^{2} \vee S^{3} \vee S^{3} \vee S^{3}$. The attaching map for the 3-cell of $T^{3}$ becomes the attaching map for the 4-cell of $\Sigma T^{3}$, a map in

$$
\pi_{3}\left(S^{2} \vee S^{2} \vee S^{2} \vee S^{3} \vee S^{3} \vee S^{3}\right) \cong \bigoplus_{1 \leq i \leq 3} \pi_{3}\left(S^{3}\right) \oplus \bigoplus_{3} \pi_{3}\left(S^{2}\right) \oplus \underset{1 \leq i<j \leq 3}{\bigoplus} \pi_{3}\left(S^{3}\right),
$$

again by the Hilton-Milnor theorem, where the last three $\pi_{3}\left(S^{3}\right)$ summands include into $\pi_{3}\left(S^{2} \vee S^{2} \vee S^{2} \vee S^{3} \vee S^{3} \vee S^{3}\right)$ via composition with the Whitehead product: let $f_{i}: S^{2} \rightarrow S_{i}^{2}$ be a generator of $\pi_{2}\left(S_{i}^{2}\right)$, where $S_{i}^{2}$ is the $i$-th $S^{2}$ component in the wedge. Then the Whitehead product is the homotopy class of the map $\left[f_{i}, f_{j}\right] \in \pi_{3}\left(S_{i}^{2} \vee S_{j}^{2}\right)$, which is the attaching map for the 4-cell in a standard cellular decomposition of $S^{2} \times S^{2}$. Since $\pi_{2}\left(S^{1}\right) \cong \pi_{2}\left(S^{1} \vee S^{1}\right) \cong 0$, the summands associated to the $S^{2}$ components of the wedge do not arise from a suspension. The summands associated to the $S^{3}$ components are null-homotopic since the 3-cell of $T^{3}$ is attached to each 2-cell twice, once on either side. This completes the explanation of the claimed homotopy equivalence:

$$
\Sigma T^{3} \simeq S^{2} \vee S^{2} \vee S^{2} \vee S^{3} \vee S^{3} \vee S^{3} \vee S^{4}
$$

By Mayer-Vietoris, the bordism group $\widetilde{\Omega}_{4}^{\mathrm{fr}}\left(S^{2} \vee S^{2} \vee S^{2} \vee S^{3} \vee S^{3} \vee S^{3} \vee S^{4}\right)$ is a direct sum

$$
\begin{aligned}
\bigoplus_{3} \widetilde{\Omega}_{4}^{\mathrm{fr}}\left(S^{2}\right) \oplus \bigoplus_{3} \widetilde{\Omega}_{4}^{\mathrm{fr}}\left(S^{3}\right) \oplus \widetilde{\Omega}_{4}^{\mathrm{fr}}\left(S^{4}\right) & \cong \bigoplus_{3} \widetilde{\Omega}_{2}^{\mathrm{fr}}\left(S^{0}\right) \oplus \bigoplus_{3} \widetilde{\Omega}_{1}^{\mathrm{fr}}\left(S^{0}\right) \oplus \widetilde{\Omega}_{0}^{\mathrm{fr}}\left(S^{0}\right) \\
& \cong \bigoplus_{3} \Omega_{2}^{\mathrm{fr}} \oplus \bigoplus_{3} \Omega_{1}^{\mathrm{fr}} \oplus \Omega_{0}^{\mathrm{fr}} \\
& \cong \bigoplus_{3} \mathbb{Z}_{2} \oplus \bigoplus_{3} \mathbb{Z}_{2} \oplus \mathbb{Z} .
\end{aligned}
$$

Therefore

$$
\Omega_{3}^{\mathrm{fr}}\left(T^{3}\right) \cong \Omega_{3}^{\mathrm{fr}} \oplus \bigoplus_{3} \mathbb{Z}_{2} \oplus \bigoplus_{3} \mathbb{Z}_{2} \oplus \mathbb{Z} \cong \mathbb{Z}_{24} \oplus \bigoplus_{3} \mathbb{Z}_{2} \oplus \bigoplus_{3} \mathbb{Z}_{2} \oplus \mathbb{Z} .
$$

The isomorphism is given as follows. Let

$$
\operatorname{pr}_{i}: T^{3}=S^{1} \times S^{1} \times S^{1} \longrightarrow S^{1}
$$


be given by projection onto the $i$-th factor. Similarly, let

$$
\mathrm{qr}_{i}: T^{3}=S^{1} \times S^{1} \times S^{1} \longrightarrow S^{1} \times S^{1}
$$

be given by forgetting the $i$-th factor. Let $F: M \rightarrow T^{3}$ be an element of $\Omega_{3}^{\mathrm{fr}}\left(T^{3}\right)$. Making all maps transverse to a point, we obtain an 8-tuple

$$
\begin{aligned}
& \left([M],\left(\mathrm{pr}_{1} \circ F\right)^{-1}(*),\left(\mathrm{pr}_{2} \circ F\right)^{-1}(*),\left(\mathrm{pr}_{3} \circ F\right)^{-1}(*),\right. \\
& \left.\left(\mathrm{qr}_{1} \circ F\right)^{-1}(*),\left(\mathrm{qr}_{2} \circ F\right)^{-1}(*),\left(\mathrm{qr}_{3} \circ F\right)^{-1}(*), F^{-1}(*)\right) \\
& \quad \in \Omega_{3}^{\mathrm{fr}} \oplus \bigoplus_{3} \Omega_{2}^{\mathrm{fr}} \oplus \bigoplus_{3} \Omega_{1}^{\mathrm{fr}} \oplus \Omega_{0}^{\mathrm{fr}} \cong \mathbb{Z}_{24} \oplus \bigoplus_{3} \mathbb{Z}_{2} \oplus \bigoplus_{3} \mathbb{Z}_{2} \oplus \mathbb{Z} .
\end{aligned}
$$

We consider each of the summands in turn.

By choosing the appropriate orientation on $M_{L}$ and making the degree-one normal maps transverse to a point, one can arrange for the disjoint union $f^{-1}(*) \sqcup-\mathrm{Id}^{-1}(*)$ to be equal to $\{\mathrm{pt}\} \sqcup-\{\mathrm{pt}\}=0 \in \Omega_{0}^{\mathrm{fr}}$.

As observed in [Davis 2006, proof of the lemma], we can change the framing so that the elements of $\Omega_{1}^{\mathrm{fr}}$ agree. First, we change the framing on each of three chosen meridians $\mu_{i}$ to the link components $L_{i}$.

Orientable $k$-plane vector bundles over $S^{1}$ are classified by homotopy classes of maps $\left[S^{1}, \mathrm{BSO}(k)\right]$. Consider the exact sequence

$$
\pi_{2}(\mathrm{BSO}) \longrightarrow \pi_{2}(\mathrm{BSO}, \mathrm{BSO}(k)) \longrightarrow \pi_{1}(\mathrm{BSO}(k)) \stackrel{\gamma}{\longrightarrow} \pi_{1}(\mathrm{BSO}) .
$$

A stably trivial vector bundle over $S^{1}$ gives us an element of $\operatorname{ker}(\gamma)$. A choice of trivialisation of the vector bundle gives us a null homotopy and therefore an element of $\pi_{2}(\mathrm{BSO}, \mathrm{BSO}(k))$. The possible choices of stable trivialisations, or framings, are indexed by $\pi_{2}(\mathrm{BSO}) \cong \pi_{1}(\mathrm{SO}) \cong \mathbb{Z}_{2}$.

We can therefore, if necessary, change the framing on each $\mu_{i}$ to be the bounding framing using an element of $\pi_{1}(\mathrm{SO}(2))$ which maps to the nontrivial element of $\pi_{1}(\mathrm{SO})$. Use the element of $\pi_{1}(\mathrm{SO}(2))$ to change the framing on the normal bundle of $\mu_{i}$ in $M_{L}$. We claim that these changes in the framing can be extended to the whole of $M_{L}$. To see this, we argue as follows. The dual of the inclusion map $H^{1}\left(M_{L} ; \mathbb{Z}\right) \rightarrow H^{1}\left(\mu_{i} ; \mathbb{Z}\right)$ is surjective, since each $\left[\mu_{i}\right]$ is a generator of $H_{1}\left(M_{L} ; \mathbb{Z}\right)$. The change of framing map $\mu_{i} \rightarrow \mathrm{SO}(2)$ represents a homotopy class of maps in $\left[\mu_{i}, S^{1}\right]$ and therefore an element of $H^{1}\left(\mu_{i} ; \mathbb{Z}\right)$. Since this pulls back to an element of $H^{1}\left(M_{L} ; \mathbb{Z}\right)$, which can in turn produce a map $M_{L} \rightarrow \mathrm{SO}(2)$, the change of framing map can be extended as claimed.

Let $N_{i} \subset M_{L}$ be the submanifolds given by $\left(\mathrm{qr}_{i} \circ f\right)^{-1}(*)$, after perturbing $f$ to make $\mathrm{qr}_{i} \circ f$ transverse to a point. As the inverse image of the $i$-th $S^{1}$ factor of $T^{3}$ (e.g., $\left.f^{-1}\left(S^{1} \times\{*\} \times\{*\}\right)\right), N_{i}$ is a collection of circles. After a homotopy of $f$, it can be arranged, by the assumption on $f$, that $N_{i}$ is a single meridian $\mu_{i}$, which has 
the bounding framing and therefore represents the zero element in $\Omega_{1}^{\mathrm{fr}}$. To make this arrangement, it suffices to be able to remove circles $N_{i}$ whose image in $T^{3}$ is null-homologous. But in $T^{3}$, a null-homologous curve is also null-homotopic. Therefore we can make a homotopy of $f$ so that $N_{i}$ misses $S^{1} \times\{*\} \times\{*\}$.

After another homotopy, the inverse image $\left(\operatorname{pr}_{i} \circ f\right)^{-1}(*)$ can be arranged to be a capped-off Seifert surface $F_{i} \cup D^{2}$, where $F_{i}$ is a Seifert surface for $L_{i}$ (possibly with closed connected components). To see this, we again use our assumption that $f$ sends the $i$-th meridian $\mu_{i}$ to the $i$-th circle. This assumption enables us to homotope $f$ so that $\mathrm{pr}_{i} \circ f$ sends a regular neighbourhood $\mu_{i} \times D^{2}$ to $S^{1}$ by projection onto the first factor. Then the inverse image is as desired. A homotopy of $f$ preserves the framed bordism class of $\left(\operatorname{pr}_{i} \circ f\right)^{-1}(*)$, and the class $\left[F_{i} \cup D^{2}\right] \in$ $\Omega_{2}^{\mathrm{fr}}$ is determined by the Arf invariant of $L_{i}$. By hypothesis, this vanishes.

Finally, again following [Davis 2006] (see also [Freedman and Quinn 1990, proof of Lemma 11.6B]), the framing can be altered in the neighbourhood of a point to change the element $[M] \in \Omega_{3}^{\text {fr }}$ to the trivial element. We recall the definition of the $J$-homomorphism $J: \pi_{3}(\mathrm{SO}) \rightarrow \pi_{3}^{S} \cong \Omega_{3}^{\mathrm{fr}}$, for the convenience of the reader, where $\pi_{k}^{S}$ is the $k$-th stable homotopy group of spheres. (Incidentally, $\pi_{3}(\mathrm{SO}) \cong \mathbb{Z}$ and $\pi_{3}^{S} \cong \mathbb{Z}_{24}$.) Given $\theta: S^{3} \rightarrow$ SO, choose a $k$ sufficiently large so that we can represent $\theta$ by a map $\theta: S^{3} \rightarrow \mathrm{SO}(k)$. We proceed to construct a map $\left(J(\theta): S^{k+3} \rightarrow S^{k}\right) \in \pi_{3}^{S}$. So:

$$
S^{k+3}=S^{3} \times D^{k} \cup_{S^{3} \times D^{k-1}} D^{4} \times S^{k-1} .
$$

Define a map

$$
\begin{aligned}
j(\theta): S^{3} \times D^{k} & \rightarrow S^{3} \times D^{k} \\
(x, y) & \mapsto(x, \theta(x)(y)),
\end{aligned}
$$

since $\theta(x) \in \mathrm{SO}(k)$ acts on $D^{k}$ by identifying $D^{k}$ with the unit ball in $\mathbb{R}^{k}$. This map extends to a homeomorphism $j(\theta)$ of $S^{3} \times D^{k} \cup_{S^{3} \times D^{k-1}} D^{4} \times S^{k-1}$. Form the composition

$$
\begin{aligned}
S^{k+3}=S^{3} \times D^{k} \cup_{S^{3} \times D^{k-1}} D^{4} \times S^{k-1} \stackrel{j(\theta)}{\longrightarrow} S^{3} \times D^{k} \cup_{S^{3} \times D^{k-1}} D^{4} \times S^{k-1} \\
\stackrel{\text { col }}{\longrightarrow} S^{3} \times S^{k} \stackrel{\text { proj }_{1}}{\longrightarrow} S^{k},
\end{aligned}
$$

where col is the collapse map which squashes $D^{4} \times S^{k-1}$ and proj $_{1}$ is the projection onto the first factor. This gives an element of $\pi_{3}^{S}$, which is the image of $\theta$ under $J: \pi_{3}(\mathrm{SO}) \rightarrow \pi_{3}^{S} \cong \Omega_{3}^{\mathrm{fr}}$.

This $J$-homomorphism is onto [Adams 1966, Example 7.17], so that composing the framing in a neighbourhood $D^{3}$ of a point with the choice of map $\theta \in \pi_{3}(\mathrm{SO})=$ $\left[\left(D^{3}, \partial D^{3}\right),(\mathrm{SO}, *)\right]$ such that $-J(\theta)=[M] \in \Omega_{3}^{\mathrm{fr}}$ changes the class in $\Omega_{3}^{\mathrm{fr}}$ as desired. 
This shows the existence of a normal cobordism $W^{\prime}$. To see that this is of degree one, note that the map to $T^{3}$ which extends over $W^{\prime}$ can be used to define a map to $T^{3} \times I$, by defining a map $g: W^{\prime} \rightarrow I$ such that $g\left(M_{L}\right)=\{0\}$ and $g\left(T^{3}\right)=\{1\}$. Now consider the commutative diagram

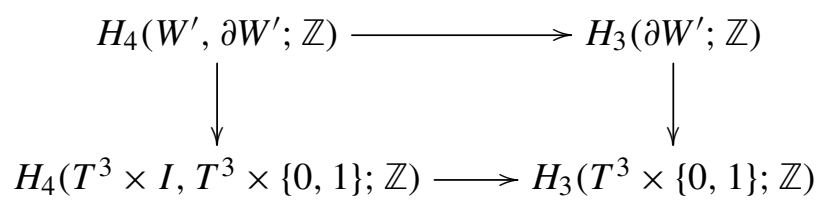

Going right, then down, the fundamental class $\left[W^{\prime}, \partial W^{\prime}\right]$ maps to

$$
(1,-1) \in H_{3}\left(T^{3} \times\{0,1\} ; \mathbb{Z}\right) \cong \mathbb{Z} \oplus \mathbb{Z} .
$$

By commutativity, the relative fundamental class $\left[W^{\prime}, \partial W^{\prime}\right]$ must map to a generator of $H_{4}\left(T^{3} \times I, T^{3} \times\{0,1\} ; \mathbb{Z}\right)$.

A $\Lambda$-homology equivalence is also an integral homology equivalence, by the following argument. By definition (see above the statement of Theorem 1.3), a $\Lambda$-homology equivalence induces an isomorphism on $H_{1}(-; \mathbb{Z})$. By duality, we also have an isomorphism on $H_{2}(-; \mathbb{Z})$. It remains to see that $f: M_{L} \rightarrow T^{3}$ is a degree-one map. The assumption that

$$
f_{*}: H_{*}\left(M_{L} ; \Lambda\right) \stackrel{\simeq}{\longrightarrow} H_{*}\left(T^{3} ; \Lambda\right)
$$

is an isomorphism implies that the relative homology vanishes: $H_{*}\left(T^{3}, M_{L} ; \Lambda\right) \cong 0$. The universal coefficient spectral sequence then implies that $H_{*}\left(T^{3}, M_{L} ; \mathbb{Z}\right) \cong 0$ since all the $E^{2}$ terms $\operatorname{Tor}_{p}^{\Lambda}\left(H_{q}\left(T^{3}, M_{L} ; \Lambda\right), \mathbb{Z}\right)$ vanish. Therefore a $\Lambda$-homology equivalence as in Theorem 1.3 is a degree-one map.

Lemma 3.4 then establishes the existence of a choice of stable framing $b$ on $M_{L}$ such that there is a degree-one normal cobordism

$$
\left(F^{\prime}: W^{\prime} \longrightarrow T^{3} \times I, e^{\prime}\right)
$$

between $\left(f: M_{L} \rightarrow T^{3}, b\right)$ and (Id : $\left.T^{3} \rightarrow T^{3}, c\right)$. Choosing such a framing, we proceed to apply surgery theory to alter $W^{\prime}$ into a homology cobordism. Davis' observation [2006] was that the framing on $W^{\prime}$ is not an intrinsic part of the concordance problem, but rather necessary additional data which is required in order to be able to apply surgery theory. Without the information provided by the self-intersection form, it is not possible to obtain algebraic sufficient conditions which ensure that surgery can be performed. Nevertheless, as we shall see, there is a certain amount of freedom in the choice of framing data.

Before giving the proof of Theorem 1.3, we first give the definition of the Wall even-dimensional surgery obstruction groups, which we will use in the proof. 
Definition 3.5 [Wall 1999, Chapter 5]. Let $A$ be a ring with involution. A $(-1)^{k}$ Hermitian sesquilinear quadratic form on a free based $A$-module $M$ is a $(-1)^{k}$ Hermitian sesquilinear form $\lambda: M \times M \rightarrow A$ together with a quadratic enhancement. A quadratic enhancement of a form $\lambda: M \times M \rightarrow A$ is a function $\mu: M \rightarrow$ $A /\left\{a-(-1)^{k} \bar{a} \mid a \in A\right\}$ such that

(1) $\lambda(x, x)=\mu(x)+\overline{\mu(x)}$,

(2) $\mu(x+y)-\mu(x)-\mu(y)=\lambda(x, y)$,

(3) $\mu(a x)=a \mu(x) \bar{a}$,

for all $x, y \in M$ and for all $a \in A$.

A hyperbolic quadratic form is a direct sum of standard hyperbolic forms, where the standard hyperbolic form $(H, \chi, v)$ is given by

$$
\left(A \oplus A,\left(\begin{array}{cc}
0 & 1 \\
(-1)^{k} & 0
\end{array}\right), v\left((1,0)^{T}\right)=0=v\left((0,1)^{T}\right)\right) .
$$

The even-dimensional surgery obstruction group $L_{2 k}(A)$ is defined to be the Witt group of nonsingular $(-1)^{k}$-Hermitian sesquilinear quadratic forms on free based $A$ modules, where addition in the Witt group is by direct sum, and the equivalence class of the hyperbolic forms is the identity element, where the equivalence relation is as follows. Quadratic forms $(M, \lambda, \mu)$ and $\left(M^{\prime}, \lambda^{\prime}, \mu^{\prime}\right)$ are said to be equivalent if there are hyperbolic forms $(H, \chi, v)$ and $\left(H^{\prime}, \chi^{\prime}, v^{\prime}\right)$ such that there is an isomorphism of forms $(M \oplus H, \lambda \oplus \chi, \mu \oplus v) \cong\left(M^{\prime} \oplus H^{\prime}, \lambda^{\prime} \oplus \chi^{\prime}, \mu^{\prime} \oplus v^{\prime}\right)$. This completes the definition of $L_{2 k}(A)$.

For us, $A$ will be the group ring $\mathbb{Z}[\pi]$ of some group $\pi$; initially $\pi$ will be $\mathbb{Z}^{3}$, so that we take $A=\mathbb{Z}\left[\mathbb{Z}^{3}\right]=\Lambda$. We omit the definition of the odd-dimensional $L$-groups since they will only play a peripheral rôle in the proof of Theorem 1.3.

Proof of Theorem 1.3. First, do surgery below the middle dimension [ibid., Chapter 1] on $\left(W^{\prime}, F^{\prime}, e^{\prime}\right)$ to create a normal cobordism $\left(F: W \rightarrow T^{3} \times I, e\right)$ which is 2-connected, i.e., $W$ is connected and $\pi_{1}(W) \cong \pi_{1}\left(T^{3}\right) \cong \mathbb{Z}^{3}$. The induced map $F_{*}: \pi_{2}(W) \rightarrow \pi_{2}\left(T^{3} \times I\right)$ is automatically surjective since $T^{3}$ is aspherical.

The Wall surgery obstruction [ibid., Chapter 5] of the normal cobordism ( $F$ : $\left.W \rightarrow T^{3} \times I, e\right)$ is now defined in $L_{4}\left(\mathbb{Z}\left[\mathbb{Z}^{3}\right]\right)$ to be given by the intersection form

$$
\lambda_{W^{\prime}}: H_{2}\left(W^{\prime} ; \Lambda\right) \times H_{2}\left(W^{\prime} ; \Lambda\right) \longrightarrow \Lambda,
$$

together with the quadratic enhancement

$$
\mu: H_{2}(W ; \Lambda) \longrightarrow \mathbb{Z}\left[\mathbb{Z}^{3}\right] /\left\{a=\bar{a} \mid a \in \mathbb{Z}\left[\mathbb{Z}^{3}\right]\right\}
$$

defined by counting the self-intersections of an immersion of a sphere $S^{2} \rightarrow W$ representing an element of $H_{2}(W ; \Lambda) \cong \pi_{2}(W)$, where the regular homotopy class 
of the immersion is fixed by the framing $e$ to be the unique class of immersions for which the induced trivialisation of $T S^{2}$ extends over the null-homotopy of $S^{2}$ in $T^{3}$.

The fact that the homology of the boundary $H_{j}\left(M_{L} ; \Lambda\right) \cong H_{j}\left(T^{3} ; \Lambda\right)$ vanishes for $j=1,2$, is used crucially here to see that the intersection form $\lambda_{W}$ is nonsingular, as observed by the surgeon in the "dialogue" of [Davis 2006].

By [Wall 1999, Proposition 13B.8], which is based on Shaneson's formula $L_{n}(\mathbb{Z}[\pi \times \mathbb{Z}]) \cong L_{n}(\mathbb{Z}[\pi]) \oplus L_{n-1}(\mathbb{Z}[\pi])$, when $\pi$ has trivial Whitehead group [Shaneson 1969] we have that

$$
\begin{aligned}
L_{4}\left(\mathbb{Z}\left[\mathbb{Z}^{3}\right]\right) & \cong \bigoplus_{i=0}^{3}\left(\begin{array}{l}
3 \\
i
\end{array}\right) L_{4-i}(\mathbb{Z}) \cong L_{4}(\mathbb{Z}) \oplus \bigoplus_{3} L_{3}(\mathbb{Z}) \oplus \bigoplus_{3} L_{2}(\mathbb{Z}) \oplus L_{1}(\mathbb{Z}) \\
& \cong L_{0}(\mathbb{Z}) \oplus \bigoplus_{3} L_{2}(\mathbb{Z}),
\end{aligned}
$$

where the last isomorphism is by periodicity of the $L$-groups and the fact that the odd-dimensional simply connected $L$-groups vanish. The even-dimensional simply connected $L$-groups $L_{2 k}(\mathbb{Z})$ are computed [Kervaire and Milnor 1963], when $k=0 \bmod 2$, as

$$
\begin{aligned}
L_{0}(\mathbb{Z}) & \stackrel{\simeq}{\rightarrow} \mathbb{Z} \\
(M, \lambda, \mu) & \mapsto \sigma\left(\mathbb{R} \otimes_{\mathbb{Z}} M, \operatorname{Id} \otimes \lambda\right) / 8,
\end{aligned}
$$

while for the dimensions where $k=1 \bmod 2$ they are computed via

$$
\begin{aligned}
L_{2}(\mathbb{Z}) & \stackrel{\simeq}{\rightarrow} \mathbb{Z}_{2} \\
(M, \lambda, \mu) & \mapsto \operatorname{Arf}\left(\mathbb{Z}_{2} \otimes_{\mathbb{Z}} M, \operatorname{Id} \otimes \lambda, \operatorname{Id} \otimes \mu\right) .
\end{aligned}
$$

We need to see that we can make further alterations to $W$ in order to make the surgery obstruction vanish.

First, we take the connected sum with $-\sigma(W) / 8$ copies of the $E_{8}$ manifold, namely the simply connected 4-manifold which is constructed by plumbing disc bundles $D^{2} \times D^{2}$ according to the $E_{8}$ lattice. It turns out that the boundary of the resulting 4-manifold is the Poincare homology sphere. One then caps off with the contractible topological 4-manifold whose boundary is the Poincare homology sphere [Freedman and Quinn 1990, Corollary 9.3C]. This produces the $E_{8}$ manifold, a closed topological 4-manifold. It has a nonsingular intersection form, with a quadratic enhancement induced from a normal map to $S^{4}$, and its signature is 8 . By a negative copy of this 4-manifold we of course mean the same manifold but with the opposite choice of orientation. By making such a modification to $W$, we obtain a new normal map, which by abuse of notation we again denote by $(W, F, e)$, for which the obstruction in $L_{0}(\mathbb{Z})$ is trivial. Note that $W$ still has fundamental group $\mathbb{Z}^{3}$ since $\pi_{1}\left(E_{8}\right.$ manifold $) \cong\{1\}$, and moreover $\partial W$ is unchanged. 
Next, we may need to alter $W$ again, so that the three Arf invariant obstructions in $L_{2}(\mathbb{Z})$ vanish. For $i=1,2,3$, define maps

$$
\mathrm{qr}_{i}: T^{3} \times I=S^{1} \times S^{1} \times S^{1} \times I \longrightarrow S^{1} \times S^{1}
$$

which forget the $i$-th $S^{1}$ factor and the $I$ factor. Perform a homotopy of $F$ to ensure that $\mathrm{qr}_{i} \circ F$ is transverse to $* \in S^{1} \times S^{1}$, and such that

$$
F^{-1}\left(S^{1} \times\{*\} \times\{*\} \times\{\partial I\}\right) \longrightarrow S^{1} \times\{*\} \times\{*\} \times\{\partial I\}
$$

is a homotopy equivalence (and similarly with the $*$ terms moved appropriately for $i=2,3$ ). This homotopy equivalence was already arranged in the proof of Lemma 3.4, when we saw that the elements of $\Omega_{1}^{\text {fr }}$ can be removed. Let $S_{i}$ be the surfaces $\left(\mathrm{qr}_{i} \circ F\right)^{-1}(*)$; each surface has boundary $\partial S_{i}$ given by the meridian $\mu_{i}$ and the corresponding $S^{1}$ factor of $T^{3}$.

Let $\mathrm{pr}_{i}: T^{3} \times I=S^{1} \times S^{1} \times S^{1} \times I \rightarrow S^{1} \times I$ be the map which remembers the $i$-th $S^{1}$ factor and the $I$ factor. Making $F$ transverse to a point, $\left(\mathrm{pr}_{i} \circ F\right)^{-1}(*)$ is a surface $\Sigma_{i} \subset W$. Since $F\left(S_{i} \cap \Sigma_{i}\right)$ is a single point and $F$ is of degree one, we can assume that $S_{i}$ and $\Sigma_{i}$ intersect in a single point. By choosing different points in the $I$ factor, we can ensure that the $\Sigma_{i}$ are all distinct.

Now, as in [Davis 2006], for each $i$ with nonzero-surgery obstruction in the corresponding $L_{2}(\mathbb{Z})$ summand of $L_{4}\left(\mathbb{Z}\left[\mathbb{Z}^{3}\right]\right)$, remove a neighbourhood $\Sigma_{i} \times D^{2}$ of $\Sigma_{i}$ and replace it with $\Sigma_{i} \times \operatorname{cl}\left(S^{1} \times S^{1} \backslash D^{2}\right)$. That is, replace the $D^{2}$ factor with a punctured torus, but define the framing on the torus to be the framing which yields Arf invariant one, that is, the Lie framing on both $S^{1}$ factors. Since $\Sigma_{i}$ is dual to $S_{i}$, this adds one to the Arf invariant of the element of $L_{2}(\mathbb{Z})$ represented by $S_{i}$, and so changes the Arf invariant one summands to having Arf invariant zero.

After these alterations we have a normal map $\left(G^{\prime}: V^{\prime} \rightarrow T^{3} \times I, k^{\prime}\right)$, with vanishing surgery obstruction. Since the fundamental group $\mathbb{Z}^{3}$ is good in the sense of Freedman (polycyclic groups are good [Freedman and Quinn 1990, Theorem 5.1A]), the surgery sequence is exact in the topological category - see [ibid., Theorem 11.3A]. We can therefore find embedded two-spheres representing a half-basis for $\pi_{2}\left(G^{\prime}\right)$, perform surgery, and obtain a topological 4-manifold $V$ which is homotopy equivalent to $T^{3} \times I$; in particular, $V$ is a homology cobordism between $M_{L}$ and $T^{3}$.

Moreover, the following diagram commutes:

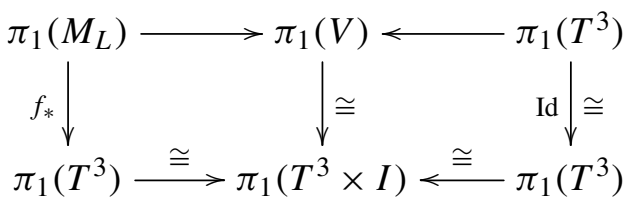


Since the meridians $\mu_{i}$ of $L$ are mapped to standard generators of $\pi_{1}\left(T^{3}\right)$, an easy diagram chase shows that the homotopy classes of the meridians are preserved in the homology cobordism $V$.

\section{Construction of links and grope concordance}

In this section we give constructions of certain links with a given Milnor invariant, and construct grope concordances, using the methods of [Cochran 1990] and [Cha 2014].

4A. Iterated Bing doubles with a prescribed Milnor invariant. Let $I$ be a multiindex with nonrepeating indices with length $m:=|I| \geq 2$. We describe a rooted binary tree $T(m)$ associated to $m \geq 2$, which has $m$ leaves: the right subtree of the root just consists of a single vertex, and the left subtree $T^{\dagger}(m)$ is the complete binary tree of height $h(m):=\left\lceil\log _{2}(m-1)\right\rceil$ with the rightmost $2(m-h(m)-1)$ pairs of leaves (and edges ending at these) removed. (By convention, a binary tree is always embedded in a plane with the root on the top.) That is, $T^{\dagger}(m)$ is a minimal height binary tree with $m-1$ leaves. For example, $T(m)$ for $m=7$ is shown in Figure 1.

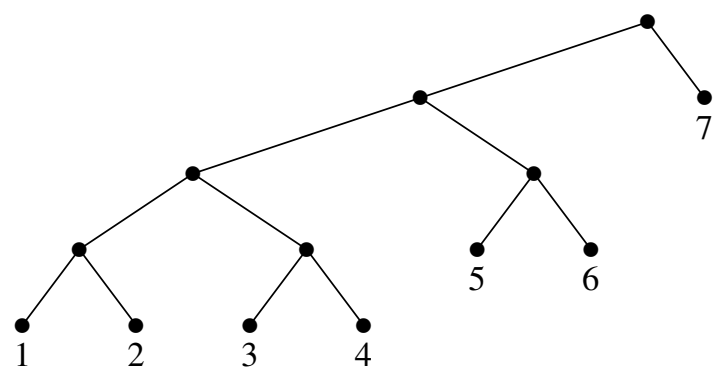

Figure 1. The tree $T(m)$ for $m=7$, labelled with $I=1234567$.

Following the proof of [Cochran 1990, Theorem 7.2], a rooted binary tree $T$ describes a link with components corresponding to the leaves of $T$. First, a complete binary tree of height one is associated to a Hopf link. If $T$ is obtained from $T^{\prime}$ by attaching two new leaves to a leaf $v$ of $T^{\prime}$, then the link associated to $T$ is obtained from that of $T$ by Bing doubling the component corresponding to $v$.

Consider the link described by the tree $T(m)$. Labelling the leaves of $T(m)$ from left to right with the multi-index $I$ (see Figure 1 for $I=1234567$ ), the components of the link are ordered. We denote this ordered link by $L_{I}$. Then, by [Cochran 1990, Theorem 8.1], the link $L_{I}$ has $\bar{\mu}_{L_{I}}(I)= \pm 1$ and $\bar{\mu}_{L_{I}}\left(I^{\prime}\right)=0$ for $\left|I^{\prime}\right|<|I|$.

4B. Satellite construction and grope concordance of links. To construct links which are grope concordant, we employ the method of [Cha 2014, Section 4]. We 
begin by giving the definition of grope concordance. The use of gropes in this context first appeared in [Cochran et al. 2003].

Definition 4.1 [Freedman and Teichner 1995]. A grope is a pair (2-complex, base circle) of a certain type described below. A grope has a height $h \in \mathbb{N}$. For $h=1$ a grope is precisely a compact oriented surface $\Sigma$ with a single boundary component which is the base circle. A grope of height $h+1$ is defined inductively as follows: let $\left\{\alpha_{i} \mid i=1, \ldots, 2 \cdot\right.$ genus $\}$ be a standard symplectic basis of circles for $\Sigma$. Then a grope of height $h+1$ is formed by attaching gropes of height $h$ to each $\alpha_{i}$ along the base circles.

An annular grope is defined by replacing the bottom stage surface by a surface with two boundary components.

Definition 4.2 [Cha 2014, Definition 2.16]. Two $m$-component links $L$ and $L^{\prime}$ in $S^{3}$ are height $n$ grope concordant if there are $m$ framed annular gropes $G_{i}$ of height $n, i=1, \ldots, m$, disjointly embedded in $S^{3} \times[0,1]$, with the boundary of $G_{i}$ the zero-framed $i$-th component of $L_{i} \subset S^{3} \times\{0\}$ and $-L_{i}^{\prime} \subset S^{3} \times\{1\}$.

As mentioned in the introduction, we could also phrase our theorems in terms of Whitney towers, but for simplicity of exposition we stick to gropes. See [ibid., Section 2] for an exposition on gropes, Whitney towers, and $n$-solvable cobordisms (our Section 5 also contains a limited discussion of $n$-solvable cobordisms).

We recall that a capped grope of height $k$ is a grope of height $k$ together with 2-discs attached along each of the standard symplectic basis curves of the top-layer surfaces. The attached 2-discs are called caps, and the grope itself is called the body. We always assume that a capped grope embedded in a 4-manifold is framed.

We denote the exterior of a link $L$ by $X_{L}$. If $L$ is a link in $S^{3}, \eta$ is an unknotted circle in $S^{3}$ disjoint from $L$, and $K$ is a knot, then we denote the satellite link of $L$ with axis $\eta$ and companion $K$ by $L(\eta, K)$; this is the image of $L$ under the homeomorphism $X_{\eta} \cup_{\partial} X_{K} \stackrel{\approx}{\rightarrow} S^{3}$, where the gluing identifies the longitude of $\eta$ with the meridian of $K$, and vice versa.

Following [ibid., Definition 4.2], we call $(L, \eta)$ a satellite configuration of height $k$ if $L$ is a link in $S^{3}, \eta$ is an unknotted circle in $S^{3}$ disjoint from $L$, and the 0-linking parallel of $\eta$ in $X_{\eta}=X_{\eta} \times\{0\}$ bounds a capped grope of height $k$ embedded in $X_{\eta} \times[0,1]$ with body disjoint from $L \times[0,1]$. The caps should be embedded in $X_{\eta} \times[0,1]$ but may intersect $L \times[0,1]$.

Lemma 4.3, stated below, describes how iterated satellite constructions using satellite configurations give us grope concordant links. The setup is as follows. Fix $n$. (To obtain Theorems 1.1 and 1.2 , set $h=n+2$.) Suppose that $\left(L_{0}, \eta\right)$ is a satellite configuration of height $k \leq n$. (Later we will use the link $L_{I}$ described above as $L_{0}$.) Suppose that $\left(K_{i}, \alpha_{i}\right)$ is a satellite configuration of height one, with $K_{i}$ a slice knot, for $i=0, \ldots, n-k-1$. Let $J_{0}^{j}$ be the connected sum 
of $N_{j}$ copies of the knot described in [Cochran and Teichner 2007, Figure 3.6], where $\left\{N_{j}\right\}$ is an increasing sequence of integers which will be specified later. (Indeed, these will be given in terms of the Cheeger-Gromov bound on the $\rho$ invariants and, for the links of Theorem 1.1, in terms of the Kneser-Haken bound on the number of disjoint nonparallel incompressible surfaces. See Section 5, just before the proof of Theorem 5.3, and Section 4D, just before Lemma 4.7.) Define $J_{i}^{j}:=K_{i-1}\left(\alpha_{i-1}, J_{i-1}^{j}\right)$ inductively for $i=1, \ldots, n-k$. Finally define $L_{j}:=L_{0}\left(\eta, J_{n-k}^{j}\right)$.

Lemma 4.3 [Cha 2014, Proposition 4.7]. The link $L_{j}$ is height $n+2$ grope concordant to $L_{0}$ for all $j$.

Proof. The same as the proof of [loc. cit.], except that $L_{0}$ replaces the Hopf link in the last sentence.

The following observation on the satellite construction is useful.

Lemma 4.4. If $L^{\prime}=L(\eta, K)$ is obtained from $L$ by a satellite construction, then $L$ and $L^{\prime}$ have the same Milnor $\bar{\mu}$-invariants.

Proof. It is well known that a satellite construction $L^{\prime}=L(\eta, K)$ comes with an integral homology equivalence $f:\left(X_{L^{\prime}}, \partial X_{L^{\prime}}\right) \rightarrow\left(X_{L}, \partial X_{L}\right)$ which restricts to a homeomorphism on the boundary preserving longitudes and meridians (see, e.g., [Cha 2010, proof of Proposition 4.8; Cha and Orr 2013, Lemma 5.3]). As in [Cha et al. 2012, Lemma 2.1], by [Stallings 1965] it follows that $f$ induces an isomorphism $\pi_{1}\left(X_{L}\right) / \pi_{1}\left(X_{L}\right)_{q} \cong \pi_{1}\left(X_{L^{\prime}}\right) / \pi_{1}\left(X_{L^{\prime}}\right)_{q}$ that preserves the classes of meridians and longitudes for any $q$, and consequently $L$ and $L^{\prime}$ have identical $\bar{\mu}$-invariants.

4C. Satellite configuration of iterated Bing doubles. Now we consider again the link $L_{I}$ described in Section 4A. Recall that $k(m):=\left\lfloor\log _{2}(m-1)\right\rfloor$, where $m=|I|$. Let $\eta$ be the zero-framed longitude of the component of $L_{I}$ labelled with $m$, namely the component of the original Hopf link that is never Bing doubled in the construction of $L_{I}$.

Lemma 4.5. (1) The pair $\left(L_{I}, \eta\right)$ is a satellite configuration of height $k(m)$.

(2) The curve $\eta$ is nonzero in $\pi_{1}\left(L_{I}\right) / \pi_{1}\left(L_{I}\right)_{m}$.

(3) For any knot $K$, the link $L_{I}(\eta, K)$ has zero-surgery manifold homeomorphic to the zero-surgery manifold of $L_{I}$.

We remark that Lemma 4.5(2) will be used in Section 5.

Proof. Denote $L:=L_{I}$ for this proof.

(1) We go back to the construction of $L$, and construct the grope as we construct $L$. We begin with the Hopf link (i.e., $m=2$ ), and the curve $\eta$ as a longitude of $L_{2}$. 
We also begin with a thickened cap $D^{2} \times[-1,1]$, such that $\partial D^{2} \times\{0\}=\eta$. This intersects the other component of the Hopf link in a single point.

Every time a component $K$ is Bing doubled in the construction of $L$, we arrange that one of the clasps lies in $D^{2} \times[-1,1]$, and then replace the thickened cap that intersected $K$ with a genus-one capped surface with a single boundary component, whose body surface misses the new Bing doubled components, and such that each cap intersects one of the two new components. See Figure 2, which is somewhat reminiscent of a figure in [Freedman and Quinn 1990, Chapter 2.1].
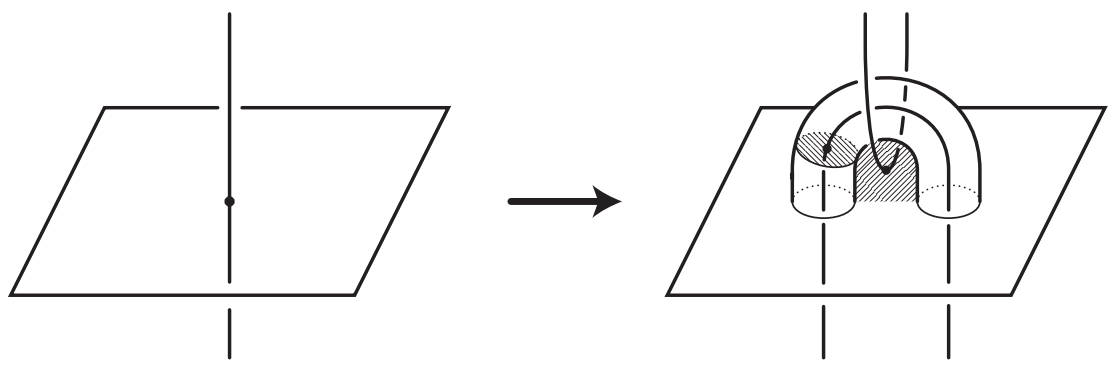

Figure 2. Replacing a cap with a capped surface.

Since a complete binary tree of height $k(m)$ can be embedded in $T(m)$, we obtain a symmetric embedded capped grope of the required height, with the body lying in the link exterior $X_{L}$ and the caps intersecting the link transversely.

(2) The nonvanishing of the Milnor invariant $\bar{\mu}_{L}(I)$ implies that all of the longitudes of $L$ are nontrivial in $\pi_{1}\left(X_{L}\right) / \pi_{1}\left(X_{L}\right)_{|I|}$.

(3) A Kirby diagram for the 3-manifold $M_{L}$ given by zero-framed surgery on $L$ can be produced by putting a 0 next to every component of $L$. If we perform a satellite construction with pattern $K$ and with $\eta$ as axis, this is equivalent to tying all the strands of $L$ which intersect a disc $D$, whose boundary is $\eta$, in the knot $K$, with framing zero. In other words, replace the trivial string link in $D \times[0,1]$ with the string link obtained by taking suitably many parallel copies of $K$.

But we can make a crossing change of these parallel copies of $K$ at will, by performing handle slides, sliding the parallel strands over the zero-framed 2-handle attached along the component parallel to $\eta$. This gives a Kirby presentation of a homeomorphic 3-manifold.

By making sufficiently many such crossing changes/handle slides, all the parallel strands which the satellite construction ties in the knot $K$ can be unknotted, recovering the link $L$. Thus the zero-surgery manifolds of the satellite link and the original link are homeomorphic. It is easy to see that the homotopy classes of the meridians of $L$ are preserved under such homeomorphisms. 
Now, let $n \geq k(|I|)=k(m)$. Let $L_{j}$ be the links obtained by the construction just before Lemma 4.3 , using our $\left(L_{I}, \eta\right)$ as $\left(L_{0}, \eta\right)$, and using the Stevedore satellite configuration described in [Cha 2014, Figure 6], which for the reader's convenience is shown in Figure 3, as the $\left(K_{i}, \alpha_{i}\right)$. Then by Lemma 4.3 and Lemma 4.5(1), the links $L_{j}$ are height $n+2$ grope concordant to the link $L_{0}=L_{I}$.

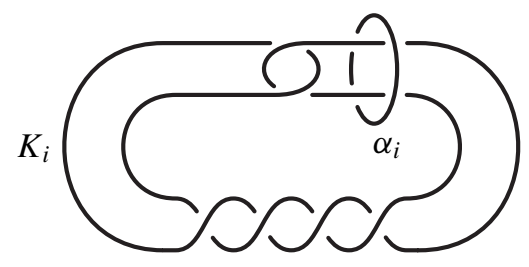

Figure 3. Stevedore satellite configuration $\left(K_{i}, \alpha_{i}\right)$.

Lemma 4.4 shows that the links $L_{j}$ satisfy Theorem 1.2(1). They also satisfy Theorem 1.2(2) by Lemma 4.5(3). We have also proved, in Lemma 4.3, the first part of Theorem 1.2(3): the links $L_{j}$ are mutually height $n+2$ grope concordant. The second part of Theorem 1.2(3), namely the failure of the links to be pairwise height $n+3$ grope concordant, will be shown in Section 5 .

4D. Examples with nonhomeomorphic zero-surgery manifolds. In order to produce examples satisfying Theorem 1.1(1), we alter the construction of Sections 4B and $4 \mathrm{C}$ to give examples with nonhomeomorphic zero-surgery manifolds. We consider the case of $m=3$ and $I=123$ only. Then the link $L:=L_{I}$ described in Section $4 \mathrm{~A}$ is the Borromean rings. Let $\eta$ be the simple closed curve in $S^{3} \backslash L$ shown in Figure $4 ; x, y$, and $z$ denote the components of $L$.

The pair $(L, \eta)$ also has two of the properties stated in Lemma 4.5, for $m=3$ :

Lemma 4.6. (1) The pair $(L, \eta)$ is a satellite configuration of height one.

(2) In $\pi_{1}\left(X_{L}\right), \eta=[x, y][[x, y], x]$, where $x, y$, and $z$ are the Wirtinger generators corresponding to the dotted arcs in Figure 4. Also, $\eta$ is nontrivial in $\pi_{1}\left(X_{L}\right) / \pi_{1}\left(X_{L}\right)_{3}$.

Here $[a, b]$ denotes the commutator $a b a^{-1} b^{-1}$.

Proof. (1) Tubing the obvious disc bounded by $\eta$ along the components of $L$ that intersect it, we obtain a genus-two surface $V$ with boundary $\eta$ which is shown in Figure 5. This is the body of the desired capped grope. The whole capped grope is the body taken together with the four caps shown in Figure 5 as shaded discs.

(2) The claim that $\eta=[x, y][[x, y], x]$ follows from a straightforward computation in terms of the Wirtinger generators, reading undercrossings of $\eta$ starting from the dot on $\eta$ in Figure 4. Since $L$ has vanishing linking number, due to Milnor [1957] 


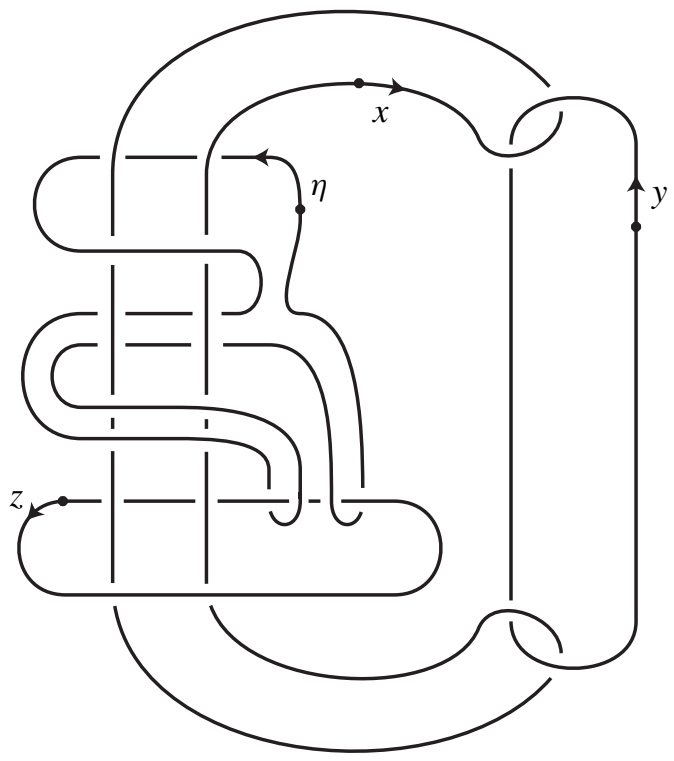

Figure 4. A satellite configuration on the Borromean rings.

(see also [Stallings 1965]), $\pi_{1}\left(X_{L}\right) / \pi_{1}\left(X_{L}\right)_{3}$ is isomorphic to $F / F_{3}$, where $F$ is the free group generated by $x, y$, and $z$. Consequently, $[[x, y], x] \in \pi_{1}\left(X_{L}\right)_{3}$ and $[x, y] \notin \pi_{1}\left(X_{L}\right)_{3}$. From this the second conclusion follows.

As in Section 4C, we apply the construction described just before Lemma 4.3, using our $(L, \eta)$ as the seed link $\left(L_{0}, \eta\right)$ and using the Stevedore satellite configuration described in [Cha 2014, Figure 6] (see our Figure 3) as $\left(K_{i}, \alpha_{i}\right)$ for $i=0, \ldots, n-2$ as above. Let the resulting links be the $L_{j}$. Then by Lemma 4.3, the $L_{j}$ are height

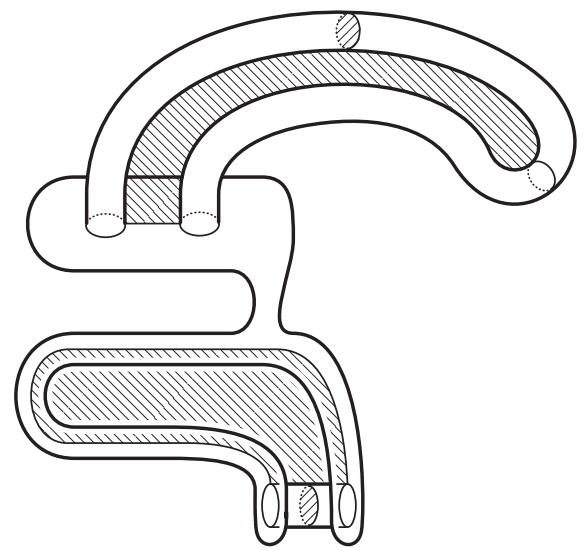

Figure 5. The capped grope bounded by $\eta$. 
$n+2$ grope concordant to the Borromean rings $L$, so these satisfy the first part of Theorem 1.1(3). The second part of Theorem 1.1(3), on the failure of the links to be pairwise height $n+3$ grope concordant, will be shown in Section 5 .

Furthermore, the links $L_{j}$ satisfies the hypothesis of Theorem 1.3. First note that since our satellite operation does not change the knot type of the components, $L_{j}$ has unknotted components. In particular, the Arf invariants of the components vanish. Recall from the proof of Lemma 4.4 that there is a homology equivalence $f: X_{L_{j}} \rightarrow$ $X_{L_{0}}$ obtained from the satellite construction $L_{j}=L_{0}\left(\eta, J_{n-1}^{j}\right)$; indeed, $f$ is obtained by gluing the identity map of $X_{L_{0} \sqcup \eta}$ with the standard homology equivalence

$$
\left(X_{J_{n-1}^{j}}, \partial X_{J_{n-1}^{j}}\right) \longrightarrow\left(S^{1} \times D^{2}, S^{1} \times S^{1}\right)
$$

along $S^{1} \times S^{1}$. Since our curve $\eta \subset S^{3}-L_{0}$ lies in the commutator subgroup of $\pi_{1}\left(S^{3}-L_{0}\right), f$ is indeed a $\Lambda$-homology equivalence $X_{L_{j}} \rightarrow X_{L_{0}}$, by a MayerVietoris argument. Filling it in with 3 solid tori, we obtain a $\Lambda$-homology equivalence $M_{L_{j}} \rightarrow T^{3}=M_{L_{0}}$ as desired. Therefore, by applying Theorem 1.3, it follows that the links $L_{j}$ satisfy Theorem 1.1(2). We need to confirm that the $L_{j}$ satisfy Theorem 1.1(1), namely, that the $M_{L_{j}}$ are not homeomorphic. The underlying idea is as follows. Recall that $L_{j}$ is defined by a satellite construction, starting with a knot $J_{0}^{j}$. In many cases, the JSJ pieces of the exterior of $J_{0}^{j}$ become parts of the JSJ decomposition of $M_{L_{j}}$, so that the $M_{L_{j}}$ have distinct JSJ decompositions. Since a complete proof of this seems to require complicated arguments (a technical issue is that an essential torus might not be parallel to a JSJ torus, because of Seifert fibred pieces), we will present a simpler argument using only the number of incompressible tori; this is enough for our purpose.

We need the following. The Kneser-Haken finiteness theorem [Haken 1961] states that for each 3-manifold $M$, there is a bound, say $C_{K H}(M)$, on the number of disjoint pairwise nonparallel incompressible surfaces that can be embedded in $M$. Recall that the knot $J_{0}^{j}$ used in the construction of the link $L_{j}$ is a connected sum of $N_{j}$ knots, where $\left\{N_{j}\right\}$ was an increasing sequence to be specified (see the paragraph before Lemma 4.3). Here is the first requirement on the $N_{j}$ : we choose the $N_{j}$ inductively in such a way that $N_{j}>\max \left\{C_{K H}\left(M_{L_{k}}\right) \mid k=0,1, \ldots, j-1\right\}$.

Lemma 4.7. The zero-surgery manifolds $M_{L_{i}}$ and $M_{L_{j}}$ are not homeomorphic for $i \neq j$.

Proof. Recall that $M_{L_{0}}=M_{L}$ is the 3-torus $T^{3}$. Consider $Y:=M_{L} \backslash v(\eta)$, where $v(\eta)$ is an open tubular neighbourhood of $\eta$. For notational convenience, denote the exterior of $J_{n-1}^{j}$ by $X:=X_{J_{n-1}^{j}}$. The 3-manifold $M_{L_{j}}$ is obtained by glueing $Y$ and $X$ along their boundaries. Let $T=\partial Y=\partial X$ be the common boundary torus. Note that $M_{L_{0}}$ can also be described in the same way, using $J_{n-1}^{0}:=$ unknot; in this case, the torus $T$ is compressible in $M_{L_{0}}$ since $X$ is a solid torus. 
Claim. For $j \geq 1$, the torus $T$ is incompressible in $Y$.

Using the claim, we will show that the 3-manifolds $M_{L_{j}}$ are not pairwise homeomorphic. Suppose $j \geq 1$. Since the knot $J_{n-1}^{j}$ is obtained from an iterated satellite construction with the first-stage knot $J_{0}^{j}$ a connected sum of $N_{j}$ nontrivial knots, the exterior $X$ of $J_{n-1}^{j}$ has at least $N_{j}$ incompressible tori, including the boundary $T$. Since $M_{L_{j}}=Y \cup_{T} X$ and $T$ is incompressible in $Y$, it follows that there are $N_{j}$ nonparallel incompressible tori in $M_{L_{j}}$. For any $k<j$, since $N_{j}>C_{K H}\left(M_{L_{k}}\right)$, it follows that $M_{L_{j}}$ is not homeomorphic to $M_{L_{k}}$.

Now, to complete the proof, we will verify the claim. If there is an essential curve on $T$ which bounds a disc in $Y$, then it must be a zero-linking longitude, say $\eta^{\prime}$, of $\eta$, since the meridian of $\eta$ is a generator of $H_{1}(Y \backslash \eta)=\mathbb{Z}^{4}$. By the following lemma, we have a contradiction.

Lemma 4.8. The class of $\eta^{\prime}$ is nontrivial in the fundamental group $\pi_{1}(Y \backslash \eta)$.

Proof. We consider a Wirtinger presentation of $\pi_{1}(Y \backslash \eta)$ given as follows: it has 24 generators, denoted by $x_{1}, \ldots, x_{24}$, associated to arcs in Figure 4. Here $\left(x_{1}, \ldots, x_{10}\right),\left(x_{11}, x_{12}\right),\left(x_{13}, \ldots, x_{16}\right)$, and $\left(x_{17}, \ldots, x_{24}\right)$ are those associated to the arcs of the components $x, y, z$, and $\eta$, respectively. In each component, the arc with a dot on it is the first one, and other arcs are ordered along the orientation. There are 27 relators:

$$
\begin{gathered}
x_{1} x_{11} \bar{x}_{1} \bar{x}_{12}, x_{11} x_{1} \bar{x}_{11} \bar{x}_{2}, x_{2} x_{18} \bar{x}_{2} \bar{x}_{19}, x_{19} x_{2} \bar{x}_{19} \bar{x}_{3}, x_{3} x_{20} \bar{x}_{3} \bar{x}_{21}, \\
x_{3} x_{23} \bar{x}_{3} \bar{x}_{22}, x_{22} x_{4} \bar{x}_{22} \bar{x}_{3}, x_{21} x_{4} \bar{x}_{21} \bar{x}_{5}, x_{5} x_{16} \bar{x}_{5} \bar{x}_{13}, x_{13} x_{5} \bar{x}_{13} \bar{x}_{6}, \\
x_{11} x_{7} \bar{x}_{11} \bar{x}_{6}, x_{7} x_{11} \bar{x}_{7} \bar{x}_{12}, x_{13} x_{8} \bar{x}_{13} \bar{x}_{7}, x_{8} x_{16} \bar{x}_{8} \bar{x}_{15}, x_{21} x_{9} \bar{x}_{21} \bar{x}_{8}, \\
x_{22} x_{9} \bar{x}_{22} \bar{x}_{10}, x_{10} x_{23} \bar{x}_{10} \bar{x}_{24}, x_{10} x_{20} \bar{x}_{10} \bar{x}_{19}, x_{19} x_{1} \bar{x}_{19} \bar{x}_{10}, x_{1} x_{18} \bar{x}_{1} \bar{x}_{17}, \\
x_{15} x_{22} \bar{x}_{15} \bar{x}_{21}, x_{22} x_{15} \bar{x}_{22} \bar{x}_{14}, x_{24} x_{13} \bar{x}_{24} \bar{x}_{14}, x_{13} x_{24} \bar{x}_{13} \bar{x}_{17}, \\
\bar{x}_{11} \bar{x}_{19} x_{22} \bar{x}_{21} \bar{x}_{13} x_{11} x_{13} x_{21} \bar{x}_{22} x_{19}, \bar{x}_{1} x_{7}, \bar{x}_{24} x_{22} x_{8} \bar{x}_{5} .
\end{gathered}
$$

Indeed, the first 24 are the standard Wirtinger relators for the 4-component link $L \sqcup \eta$ (thus one of these may be omitted), and the last 3 relators arise from the zero-surgery performed along $L$. It is straightforward to read off the curve $\eta^{\prime}$ :

$$
\eta^{\prime}=x_{1} \bar{x}_{2} x_{10} \bar{x}_{3} x_{15} x_{3} \bar{x}_{10} \bar{x}_{13} \text {. }
$$

We define a representation $\rho: \pi_{1}(Y \backslash \eta) \rightarrow \mathrm{SL}\left(2, \mathbb{Z}_{5}\right)$ by mapping the above 24 generators, respectively, to:

$$
\begin{aligned}
& {\left[\begin{array}{ll}
0 & 4 \\
1 & 3
\end{array}\right],\left[\begin{array}{ll}
4 & 0 \\
1 & 4
\end{array}\right],\left[\begin{array}{ll}
0 & 4 \\
1 & 3
\end{array}\right],\left[\begin{array}{ll}
4 & 4 \\
0 & 4
\end{array}\right],\left[\begin{array}{ll}
2 & 1 \\
1 & 1
\end{array}\right],\left[\begin{array}{ll}
4 & 0 \\
1 & 4
\end{array}\right],\left[\begin{array}{ll}
0 & 4 \\
1 & 3
\end{array}\right],\left[\begin{array}{ll}
4 & 0 \\
4 & 4
\end{array}\right],} \\
& {\left[\begin{array}{ll}
1 & 1 \\
1 & 2
\end{array}\right],\left[\begin{array}{ll}
1 & 1 \\
1 & 2
\end{array}\right],\left[\begin{array}{ll}
4 & 1 \\
4 & 0
\end{array}\right],\left[\begin{array}{ll}
3 & 1 \\
2 & 1
\end{array}\right],\left[\begin{array}{ll}
4 & 3 \\
2 & 3
\end{array}\right],\left[\begin{array}{ll}
1 & 3 \\
0 & 1
\end{array}\right],\left[\begin{array}{ll}
2 & 2 \\
2 & 0
\end{array}\right],\left[\begin{array}{ll}
4 & 2 \\
3 & 3
\end{array}\right],} \\
& {\left[\begin{array}{ll}
0 & 1 \\
4 & 2
\end{array}\right],\left[\begin{array}{ll}
0 & 1 \\
4 & 2
\end{array}\right],\left[\begin{array}{ll}
1 & 1 \\
0 & 1
\end{array}\right],\left[\begin{array}{ll}
3 & 4 \\
4 & 4
\end{array}\right],\left[\begin{array}{ll}
2 & 1 \\
4 & 0
\end{array}\right],\left[\begin{array}{ll}
4 & 4 \\
4 & 3
\end{array}\right],\left[\begin{array}{ll}
0 & 4 \\
1 & 2
\end{array}\right],\left[\begin{array}{ll}
1 & 0 \\
1 & 1
\end{array}\right] .}
\end{aligned}
$$


It can be verified that all the relators are sent to the identity, by a straightforward computation. (We found the representation $\rho$ using a computer program.) Also, we have that

$$
\rho\left(\eta^{\prime}\right)=\left[\begin{array}{ll}
3 & 1 \\
4 & 0
\end{array}\right]
$$

is not the identity. This completes the proof.

\section{Grope concordance and amenable signatures}

In this section we show that the links described in Sections 4C and 4D are not height $n+3$ grope concordant by using amenable signature obstructions from [Cha 2014]. In fact, the amenable signatures we use are obstructions to being $n$-solvably cobordant, which is a relative analogue for manifolds with boundary, or bordered manifolds, of the notion of $n$-solvability of [Cochran et al. 2003]. For our purpose it suffices to consider the case of link exteriors; an $n$-solvable cobordism between the exteriors $X$ and $X^{\prime}$ of two links with the same number of components is a 4-manifold $W$ with $\partial W=X \cup_{\partial}-X^{\prime}$ satisfying the conditions described in [Cha 2014, Definition 2.8], where the boundary tori of $X$ and $X^{\prime}$ are identified along the zero framing. Since we do not use the defining condition right now, instead of spelling it out here, we begin with its relationship to grope concordance. The following theorem originates from [Cochran et al. 2003, Theorem 8.11], and was given in our context in [Cha 2014].

Theorem 5.1 [ibid., Theorems 2.16 and 2.13, and Remark 2.11]. If two links are height $n+2$ grope concordant, then their exteriors are $n$-solvably cobordant as bordered 3-manifolds.

As our key ingredient to detect nonsolvably cobordant 3-manifolds and therefore non-grope-concordant links, we will use the Amenable Signature Theorem, which was first introduced in [Cha and Orr 2012] for homology cobordism of closed 3-manifolds and then generalised to $n$-solvable cobordisms of bordered 3-manifolds in [Cha 2014]. We state a special case which will be sufficient for our purpose. For a closed 3-manifold $M$ and a homomorphism $\phi: \pi_{1}(M) \rightarrow G$, denote the von Neumann-Cheeger-Gromov $\rho$-invariant by $\rho^{(2)}(M, \phi) \in \mathbb{R}$. See, e.g., [Cochran et al. 2003, Section 5] as well as [Chang and Weinberger 2003; Harvey 2008; Cha 2008; Cha and Orr 2012] for definitions and useful properties of $\rho^{(2)}(M, \phi)$. Precise references for the properties that we need will be recalled as we go along.

Theorem 5.2 (A special case of [Cha 2014, Amenable Signature Theorem 3.2]). Suppose $W$ is an $(n+1)$-solvable cobordism between two bordered 3-manifolds $X$ and $X^{\prime}$, and $G$ admits a subnormal series

$$
G=G_{0} \supset G_{1} \supset \cdots \supset G_{n} \supset G_{n+1}=\{e\}
$$


with each quotient $G_{i} / G_{i+1}$ torsion-free abelian. Then $\rho^{(2)}\left(X \cup_{\partial}-X^{\prime}, \phi\right)=0$ for any $\phi: \pi_{1}\left(X \cup_{\partial}-X^{\prime}\right) \rightarrow G$ which factors through $\pi_{1}(W)$.

Recall that in our construction of the links $L_{j}$, the knot $J_{0}^{j}$ was the connected sum of $N_{j}$ copies of Cochran and Teichner's knot, say $J$. Now we proceed to specify the integers $N_{j}$. Denote by $\rho^{(2)}(K):=\int_{S^{1}} \sigma_{K}(\omega) d \omega$ the integral of the LevineTristram signature function over the circle normalised to length one. We have $\rho^{(2)}\left(J_{0}^{j}\right)=N_{j} \rho^{(2)}(J)=4 N_{j} / 3$ by additivity under connected sum and [Cochran and Teichner 2007, Lemma 4.5]. Due to Cheeger and Gromov [1985], for any closed 3-manifold $Y$ there is a constant $C_{Y}>0$ such that $\left|\rho^{(2)}(Y, \psi)\right|<C_{Y}$ for any $\psi$. From now on we abbreviate $\ell:=n-k(m)$. Define

$$
R:=C_{X_{L_{0}} \cup_{\partial}-X_{L_{0}}}+2 \sum_{i=0}^{\ell-1} C_{M_{K_{i}}} .
$$

We choose the large integers $N_{j}$ inductively in such a way that

$$
N_{j}>3 R / 4+\max \left\{N_{k} \mid k<j\right\} .
$$

Then we have

$$
\rho^{(2)}\left(J_{0}^{j}\right)>R+\rho^{(2)}\left(J_{0}^{k}\right)
$$

whenever $j>k$. For Theorem 1.1, we make these choices so that the condition in the preamble to Lemma 4.7 relating to the Kneser-Haken bound is simultaneously satisfied.

Now we start the proof that our links $L_{j}$ are not height $n+3$ grope concordant to one another. Let $X$ and $X^{\prime}$ be the exteriors of $L_{j}$ and $L_{k}$, respectively. To distinguish them in the notation, we denote the axis curve $\eta$ in $X$ by $\eta_{j}$, and we denote the corresponding axis curve in $X^{\prime}$ by $\eta_{k}$.

Recall that $m=|I|$ and that $k(m)=\left\lfloor\log _{2}(m-1)\right\rfloor$. Also note that $k(m)+1=$ $\left\lceil\log _{2}(m)\right\rceil$. By Theorem 5.1, it suffices to show the following:

Theorem 5.3. For $n \geq k(m)$, the bordered 3-manifolds $X$ and $X^{\prime}$ are not $(n+1)$ solvably cobordant when $j \neq k$.

By Theorem 5.1, it then follows that our links $L_{j}$ and $L_{k}$ are not height $n+3$ grope concordant when $j \neq k$.

Proof. The proof proceeds almost identically to that of [Cha 2014, Theorem 4.8], which combines the Amenable Signature Theorem of that reference with a higherorder Blanchfield duality argument for a certain 4-dimensional cobordism introduced in [Cochran et al. 2009] (see our $W_{0}$ below). So we will give an outline for our case and discuss differences from [Cha 2014, Theorem 4.8].

Suppose $W$ is an $(n+1)$-solvable cobordism with $\partial W=X \cup_{\partial}-X^{\prime}$. Similarly to [ibid., Section 4.3] (see the paragraph entitled "Cobordism associated to an iterated 
satellite construction"), we consider a cobordism $V$ with

$$
\begin{aligned}
\partial V=M_{J_{0}^{j}} \sqcup-M_{J_{0}^{k}} \sqcup M_{K_{0}} \sqcup-M_{K_{0}}^{\prime} \sqcup \cdots \sqcup M_{K_{\ell-1}} \sqcup-M_{K_{\ell-1}}^{\prime} \\
\qquad\left(X_{L_{0}} \cup_{\partial}-X_{L_{0}}\right) \sqcup-\left(X \cup_{\partial}-X^{\prime}\right)
\end{aligned}
$$

which is built by stacking cobordisms associated to satellite constructions [Cochran et al. 2009, p. 1429], where $M_{K_{i}}^{\prime}$ is a copy of $M_{K_{i}}$, and then construct a cobordism $W_{0}$ with

$$
\partial W_{0}=M_{J_{0}^{j}} \sqcup-M_{J_{0}^{k}} \sqcup M_{K_{0}} \sqcup-M_{K_{0}}^{\prime} \sqcup \cdots \sqcup M_{K_{\ell-1}} \sqcup-M_{K_{\ell-1}}^{\prime} \sqcup\left(X_{L_{0}} \cup_{\partial}-X_{L_{0}}\right)
$$

by attaching $V$ to $W$ along $X \cup_{\partial}-X^{\prime}$. We omit the detailed construction of $V$ and $W_{0}$ but state a couple of useful facts which can be verified as in [Cha 2014, Section 4.3]. Let $\left\{\mathscr{P}^{r} G\right\}$ be the rational derived series of a group $G$, i.e., $\mathscr{P}^{0} G:=G$ and $\mathscr{P}^{r+1} G$ is the kernel of $\mathscr{P}^{r} G \rightarrow H_{1}\left(\mathscr{P}^{r} G ; \mathbb{Q}\right)$. Let $\phi_{0}$ be the quotient map $\pi_{1}\left(W_{0}\right) \rightarrow G:=\pi_{1}\left(W_{0}\right) / \mathscr{P}^{n+1} \pi_{1}\left(W_{0}\right)$. Also we denote by $\phi_{0}$ the restrictions of $\phi_{0}$ to the components of $\partial W_{0}$ and to $W \subset W_{0}$, as an abuse of notation. Then we have the following facts:

$$
\begin{aligned}
\rho^{(2)}\left(M_{J_{0}^{j}}, \phi_{0}\right) & -\rho^{(2)}\left(M_{J_{0}^{k}}, \phi_{0}\right)+\rho^{(2)}\left(X_{L_{0}} \cup_{\partial}-X_{L_{0}}, \phi_{0}\right) \\
& +\sum_{i=0}^{\ell-1} \rho^{(2)}\left(M_{K_{i}}, \phi_{0}\right)-\sum_{i=0}^{\ell-1} \rho^{(2)}\left(M_{K_{i}}^{\prime}, \phi_{0}\right)=\rho^{(2)}\left(X \cup_{\partial}-X^{\prime}, \phi_{0}\right) .
\end{aligned}
$$

(2) The image of the meridian of $J_{0}^{j}$ in $M_{J_{0}^{j}} \subset \partial W_{0}$ under $\phi_{0}$ is a nontrivial element in the torsion-free abelian subgroup $\mathscr{P}^{n} \pi_{1}(W) / \mathscr{P}^{n+1} \pi_{1}(W)$ of $G$. Similarly for $k$ instead of $j$.

The proof of (1) is completely identical to that given in [Cha 2014, Section 4.3] (see the paragraphs entitled "Cobordism associated to an iterated satellite construction" and "Applications of Amenable Signature Theorem"): briefly, the $\rho^{(2)}$-invariant of $\partial W_{0}$, which is the left-hand side of (1), is equal to the $L^{2}$-signature defect of $W_{0}=V \cup_{X \cup_{\partial}-X^{\prime}} W$ (this is a standard fact from index theory, or can be taken as the definition of $\left.\rho^{(2)}\right)$. It turns out that $V$ has no contribution to the $L^{2}$-signature defect, by [Cochran et al. 2009, Lemma 2.4]. So the left-hand side of (1) is equal to the $L^{2}$-signature defect of $W$, which is the $\rho^{(2)}$-invariant of $\partial W$, namely the right-hand side of (1).

The proof of (2) is almost identical to that given in [Cha 2014, Theorem 4.10]. Only the following change is required: in the initial step of the inductive argument in that result, it was shown that the image of (a parallel copy of) $\eta \subset X \subset \partial W$ is nontrivial under the quotient map $\pi_{1}(W) \rightarrow \pi_{1}(W) / \mathscr{P}^{2} \pi_{1}(W)$ (see the fourth paragraph of the proof) using a Blanchfield duality argument. 
In our case, instead we use Lemma 5.4 below, which is a generalisation of [Cha et al. 2012, Lemma 3.5], to show that the image of $\eta$ is nontrivial in the quotient $\pi_{1}(W) / \mathscr{P}^{(k(m)+1)} \pi_{1}(W)$. The argument used in Lemma 5.4 is essentially an application of Dwyer's theorem.

Lemma 5.4. If $W$ is an $n$-solvable cobordism between two link exteriors (or, more generally, bordered 3-manifolds) $X$ and $X^{\prime}$, then the inclusions induce isomorphisms

$$
\pi_{1}(X) / \pi_{1}(X)_{q} \cong \pi_{1}(W) / \pi_{1}(W)_{q} \cong \pi_{1}\left(X^{\prime}\right) / \pi_{1}\left(X^{\prime}\right)_{q}
$$

for $q \leq 2^{n}+1$.

Proof. Recall Dwyer's theorem [1975]: if $f: X \rightarrow Y$ induces an isomorphism $H_{1}(X ; \mathbb{Z}) \cong H_{1}(Y ; \mathbb{Z})$ and an epimorphism

$$
H_{2}(X ; \mathbb{Z}) \longrightarrow H_{2}(Y ; \mathbb{Z}) / \operatorname{Im}\left\{H_{2}\left(Y ; \mathbb{Z}\left[\pi_{1}(W) / \pi_{1}(W)_{q}\right]\right) \rightarrow H_{2}(Y ; \mathbb{Z})\right\},
$$

then $f$ induces an isomorphism $\pi_{1}(X)_{q} / \pi_{1}(X)_{q+1} \cong \pi_{1}(Y)_{q} / \pi_{1}(Y)_{q+1}$.

In our case, by the definition of an $n$-solvable cobordism [Cha 2014, Definition 2.8], we have $H_{1}(X ; \mathbb{Z}) \cong H_{1}(W ; \mathbb{Z}) \cong H_{1}\left(X^{\prime} ; \mathbb{Z}\right)$. Also, by the same definition, there are elements $\ell_{1}, \ldots, \ell_{r}, d_{1}, \ldots, d_{r}$ lying in $H_{2}\left(W ; \mathbb{Z}\left[\pi_{1}(W) / \pi_{1}(W)^{(n)}\right]\right)$ such that the images of $\ell_{i}$ and $d_{j}$ generate $H_{2}(W ; \mathbb{Z})$. Since $\pi_{1}(W)^{(n)}$ is contained in $\pi_{1}(W)_{2^{n}}$, the $H_{2}$ condition of Dwyer's theorem is satisfied. Therefore, it follows that

$$
\pi_{1}(X)_{q} / \pi_{1}(X)_{q+1} \cong \pi_{1}(W)_{q} / \pi_{1}(W)_{q+1} \cong \pi_{1}\left(X^{\prime}\right)_{q} / \pi_{1}\left(X^{\prime}\right)_{q+1}
$$

for $q \leq 2^{n}$ by Dwyer's theorem. From this the desired conclusion follows by the five lemma.

Recall that Lemma 4.5(2) implies that $\eta \subset X$ represents a nontrivial element in $\pi_{1}(X) / \pi_{1}(X)_{m}$. Since the above isomorphisms preserve longitudes (and meridians), $\eta_{j} \subset X$ represents a nontrivial element in $\pi_{1}(W) / \pi_{1}(W)_{m}$. Since $L_{j}$ has vanishing Milnor invariants of length less than $|I|=m$, we have $\pi_{1}(X) / \pi_{1}(X)_{m} \cong F / F_{m}$, where $F$ is the free group with rank $m$, by [Milnor 1957, Theorem 4]. Consequently $\pi_{1}(W) / \pi_{1}(W)_{m}$ is torsion-free.

We note that for any group $\pi$, we have $\pi^{(k(q)+1)}=\pi^{\left(\left[\log _{2}(q)\right\rceil\right)} \subseteq \pi_{q}$. Therefore there is a quotient map $\pi_{1}(W) / \pi_{1}(W)^{(k(m)+1)} \rightarrow \pi_{1}(W) / \pi_{1}(W)_{m}$, and this map factors through $\pi_{1}(W) / \mathscr{P}^{(k(m)+1)} \pi_{1}(W)$ by the definition of $\mathscr{P}^{(k(m)+1)}$ and the fact that the codomain is torsion-free. Since $\eta_{j}$ is nontrivial in $\pi_{1}(W) / \pi_{1}(W)_{m}, \eta_{j}$ is also nontrivial in $\pi_{1}(W) / \mathscr{P}^{(k(m)+1)} \pi_{1}(W)$. By replacing $j$ with $k$ and $X$ with $X^{\prime}$ we obtain the corresponding fact for $\eta_{k}$ in $X^{\prime}$.

To complete the proof of Theorem 5.3, we proceed as in [Cha 2014, Section 4.3]. Observe that for the normal subgroups $G_{i}:=\mathscr{P}^{i} \pi_{1}\left(W_{0}\right) / \mathscr{P}^{n+1} \pi_{1}\left(W_{0}\right)$ of our $G$, the quotient $G_{i} / G_{i+1}$ is torsion-free abelian. So by Amenable Signature Theorem 5.2 we have $\rho^{(2)}\left(X \cup_{\partial}-X^{\prime}\right)=0$. Since the curve $\eta_{j}$ represents a nontrivial element 
in a torsion-free abelian normal subgroup of $G$, the image of $\pi_{1}\left(M_{J_{0}^{j}}\right)$ in $G$ under $\phi_{0}$ is the infinite cyclic group. By $L^{2}$-induction (see, e.g., [Cheeger and Gromov 1985, page 8(2.3); Cochran et al. 2003, Proposition 5.13]) and [Cochran et al. 2004, Proposition 5.1], we have $\rho^{(2)}\left(M_{J_{0}^{j}}, \phi_{0}\right)=\rho^{(2)}\left(J_{0}^{j}\right)$, and similarly for $J_{0}^{k}$. Now, combining these two facts with (1), we obtain

$$
\begin{aligned}
\rho^{(2)}\left(J_{0}^{j}\right)-\rho^{(2)}\left(J_{0}^{k}\right)+\rho^{(2)}( & \left.X_{L_{0}} \cup_{\partial}-X_{L_{0}}, \phi_{0}\right) \\
& +\sum_{i=0}^{\ell-1} \rho^{(2)}\left(M_{K_{i}}, \phi_{0}\right)-\sum_{i=0}^{\ell-1} \rho^{(2)}\left(M_{K_{i}}^{\prime}, \phi_{0}\right)=0 .
\end{aligned}
$$

Recall that

$$
\begin{aligned}
\mid \rho^{(2)}\left(X_{L_{0}} \cup_{\partial}-X_{L_{0}}, \phi_{0}\right)+\sum_{i=0}^{\ell-1} \rho^{(2)}\left(M_{K_{i}}, \phi_{0}\right)- & \sum_{i=0}^{\ell-1} \rho^{(2)}\left(M_{K_{i}}^{\prime}, \phi_{0}\right) \mid \\
& <R:=C_{X_{L_{0}} \cup_{\partial}-X_{L_{0}}}+2 \sum_{i=0}^{\ell-1} C_{M_{K_{i}}},
\end{aligned}
$$

and in the preamble to Theorem 5.3, we chose $N_{j}$ so that $\left|\rho^{(2)}\left(J_{0}^{k}\right)-\rho^{(2)}\left(J_{0}^{j}\right)\right|>R$ whenever $k \neq j$. Therefore (3) implies that $j=k$. Thus the existence of the $(n+1)$-solvable cobordism $W$ implies that $j=k$, which is the contrapositive of the desired statement.

\section{Acknowledgements}

We would like to thank Stefan Friedl for many conversations: our first examples were motivated by joint work with him. We also thank Jim Davis for discussions relating to the proof of his theorem on Hopf links, and Prudence Heck, Charles Livingston, Kent Orr and Vladimir Turaev for helpful discussions. Finally we thank the referee for valuable comments and for being impressively expeditious.

Part of this work was completed while the first author was a visitor at Indiana University in Bloomington and the second author was a visitor at the Max Planck Institute for Mathematics in Bonn. We would like to thank these institutions for their hospitality.

The first author was partially supported by National Research Foundation of Korea grants 2013067043 and 2013053914. The second author gratefully acknowledges an AMS Simons travel grant which aided his travel to Bonn.

\section{References}

[Adams 1966] J. F. Adams, "On the groups $J(X)$, IV”, Topology 5 (1966), 21-71. MR 33 \#6628 Zbl 0145.19902 
[Cappell and Shaneson 1974] S. E. Cappell and J. L. Shaneson, "The codimension two placement problem and homology equivalent manifolds", Ann. of Math. (2) 99 (1974), 277-348. MR 49 \#3978 Zbl 0279.57011

[Cappell and Shaneson 1980] S. E. Cappell and J. L. Shaneson, "Link cobordism", Comment. Math. Helv. 55:1 (1980), 20-49. MR 81j:57011 Zbl 0444.57008

[Casson and Gordon 1978] A. J. Casson and C. M. Gordon, "On slice knots in dimension three", pp. 39-53 in Algebraic and geometric topology (Stanford, CA, 1976), vol. 2, edited by R. J. Milgram, Proc. Sympos. Pure Math. 32, Amer. Math. Soc., Providence, RI, 1978. MR 81g:57003 Zbl 0394.57008

[Casson and Gordon 1986] A. J. Casson and C. M. Gordon, "Cobordism of classical knots", pp. 181-199 in À la recherche de la topologie perdue, edited by L. Guillou and A. Marin, Progr. Math. 62, Birkhäuser, Boston, 1986. MR 900252

[Cha 2008] J. C. Cha, "Topological minimal genus and $L^{2}$-signatures", Algebr. Geom. Topol. 8:2 (2008), 885-909. MR 2010f:57024 Zbl 1162.57016

[Cha 2010] J. C. Cha, "Link concordance, homology cobordism, and Hirzebruch-type defects from iterated p-covers", J. Eur. Math. Soc. (JEMS) 12:3 (2010), 555-610. MR 2011f:57003 Zbl 1195.57012

[Cha 2014] J. C. Cha, "Symmetric Whitney tower cobordism for bordered 3-manifolds and links", Trans. Amer. Math. Soc. 366:6 (2014), 3241-3273. MR 3180746

[Cha and Ko 1999] J. C. Cha and K. H. Ko, "On equivariant slice knots", Proc. Amer. Math. Soc. 127:7 (1999), 2175-2182. MR 2000a:57006 Zbl 0959.57003

[Cha and Orr 2012] J. C. Cha and K. E. Orr, " $L^{2}$-signatures, homology localization, and amenable groups”, Comm. Pure Appl. Math. 65:6 (2012), 790-832. MR 2903800 Zbl 1256.57018

[Cha and Orr 2013] J. C. Cha and K. E. Orr, "Hidden torsion, 3-manifolds, and homology cobordism", J. Topol. 6:2 (2013), 490-512. MR 3065182

[Cha et al. 2012] J. C. Cha, S. Friedl, and M. Powell, "Concordance of links with identical Alexander invariants", preprint, 2012. arXiv 1212.2924

[Chang and Weinberger 2003] S. Chang and S. Weinberger, "On invariants of Hirzebruch and Cheeger-Gromov”, Geom. Topol. 7 (2003), 311-319. MR 2004c:57052 Zbl 1037.57028

[Cheeger and Gromov 1985] J. Cheeger and M. Gromov, "Bounds on the von Neumann dimension of $L^{2}$-cohomology and the Gauss-Bonnet theorem for open manifolds", J. Differential Geom. 21:1 (1985), 1-34. MR 87d:58136 Zbl 0614.53034

[Cochran 1985] T. D. Cochran, "Concordance invariance of coefficients of Conway's link polynomial”, Invent. Math. 82:3 (1985), 527-541. MR 87c:57002 Zbl 0589.57005

[Cochran 1990] T. D. Cochran, “Derivatives of links: Milnor's concordance invariants and Massey's products", Mem. Amer. Math. Soc. 84:427 (1990). MR 91c:57005 Zbl 0705.57003

[Cochran and Kim 2008] T. D. Cochran and T. Kim, "Higher-order Alexander invariants and filtrations of the knot concordance group", Trans. Amer. Math. Soc. 360:3 (2008), 1407-1441. MR 2008m:57008 Zbl 1132.57005

[Cochran and Teichner 2007] T. D. Cochran and P. Teichner, "Knot concordance and von Neumann $\rho$-invariants”, Duke Math. J. 137:2 (2007), 337-379. MR 2008f:57005 Zbl 1186.57006

[Cochran et al. 2003] T. D. Cochran, K. E. Orr, and P. Teichner, "Knot concordance, Whitney towers and $L^{2}$-signatures", Ann. of Math. (2) 157:2 (2003), 433-519. MR 2004i:57003 Zbl 1044.57001

[Cochran et al. 2004] T. D. Cochran, K. E. Orr, and P. Teichner, "Structure in the classical knot concordance group", Comment. Math. Helv. 79:1 (2004), 105-123. MR 2004k:57005 Zbl 1061.57008 
[Cochran et al. 2009] T. D. Cochran, S. Harvey, and C. Leidy, "Knot concordance and higher-order Blanchfield duality", Geom. Topol. 13:3 (2009), 1419-1482. MR 2009m:57006 Zbl 1175.57004

[Cochran et al. 2013] T. D. Cochran, B. D. Franklin, M. Hedden, and P. D. Horn, "Knot concordance and homology cobordism", Proc. Amer. Math. Soc. 141:6 (2013), 2193-2208. MR 3034445 Zbl 1276.57007 arXiv 1102.5730

[Davis 2006] J. F. Davis, "A two component link with Alexander polynomial one is concordant to the Hopf link", Math. Proc. Cambridge Philos. Soc. 140:2 (2006), 265-268. MR 2006k:57010 Zbl 1090.57005

[Dwyer 1975] W. G. Dwyer, "Homology, Massey products and maps between groups", J. Pure Appl. Algebra 6:2 (1975), 177-190. MR 52 \#6710 Zbl 0338.20057

[Freedman and Quinn 1990] M. H. Freedman and F. Quinn, Topology of 4-manifolds, Princeton Mathematical Series 39, Princeton University Press, 1990. MR 94b:57021 Zbl 0705.57001

[Freedman and Teichner 1995] M. H. Freedman and P. Teichner, "4-manifold topology, I: Subexponential groups”, Invent. Math. 122:3 (1995), 509-529. MR 96k:57015 Zbl 0857.57017

[Friedl and Powell 2011] S. Friedl and M. Powell, "Links not concordant to the Hopf link", Math. Proc. Cambridge Philos. Soc. (2011). To appear. arXiv 1105.2773

[Friedl and Teichner 2005] S. Friedl and P. Teichner, "New topologically slice knots", Geom. Topol. 9 (2005), 2129-2158. MR 2007b:57007 Zbl 1120.57004

[Haken 1961] W. Haken, "Ein Verfahren zur Aufspaltung einer 3-Mannigfaltigkeit in irreduzible 3-Mannigfaltigkeiten”, Math. Z. 76 (1961), 427-467. MR 25 \#4519c Zbl 0111.18803

[Harvey 2008] S. L. Harvey, "Homology cobordism invariants and the Cochran-Orr-Teichner filtration of the link concordance group", Geom. Topol. 12:1 (2008), 387-430. MR 2009d:57045 Zbl 1157.57006

[Hillman 2002] J. Hillman, Algebraic invariants of links, Series on Knots and Everything 32, World Scientific, River Edge, NJ, 2002. MR 2003k:57014 Zbl 1007.57001

[Hilton 1955] P. J. Hilton, “On the homotopy groups of the union of spheres", J. London Math. Soc. 30 (1955), 154-172. MR 16,847d Zbl 0064.17301

[Kawauchi 1978] A. Kawauchi, "On the Alexander polynomials of cobordant links", Osaka J. Math. 15:1 (1978), 151-159. MR 58 \#7599 Zbl 0401.57013

[Kawauchi 1996] A. Kawauchi, A survey of knot theory, Birkhäuser, Basel, 1996. MR 97k:57011 Zbl 0861.57001

[Kervaire and Milnor 1963] M. A. Kervaire and J. W. Milnor, "Groups of homotopy spheres, I", Ann. of Math. (2) 77 (1963), 504-537. MR 26 \#5584 Zbl 0115.40505

[Le Dimet 1988] J.-Y. Le Dimet, Cobordisme d'enlacements de disques, Mém. Soc. Math. France (N.S.) 32, Soc. Math. France, Paris, 1988. MR 90e:57046 Zbl 0666.57015

[Levine 1966] J. Levine, "Polynomial invariants of knots of codimension two", Ann. of Math. (2) 84 (1966), 537-554. MR 34 \#808 Zbl 0196.55905

[Levine 1969a] J. Levine, "Invariants of knot cobordism", Invent. Math. 8 (1969), 98-110, 355. MR 40 \#6563 Zbl 0179.52401

[Levine 1969b] J. Levine, "Knot cobordism groups in codimension two", Comment. Math. Helv. 44 (1969), 229-244. MR 39 \#7618 Zbl 0176.22101

[Levine 1977] J. Levine, "Knot modules, I”, Trans. Amer. Math. Soc. 229 (1977), 1-50. MR 57 \#1503 Zbl 0653.57012

[Livingston 1983] C. Livingston, "Knots which are not concordant to their reverses", Quart. J. Math. Oxford Ser. (2) 34:135 (1983), 323-328. MR 85d:57005 Zbl 0537.57003 
[Milnor 1957] J. Milnor, "Isotopy of links", pp. 280-306 in Algebraic geometry and topology: a symposium in honor of S. Lefschetz, edited by R. H. Fox et al., Princeton Math. Series 12, Princeton University Press, 1957. MR 19,1070c Zbl 0080.16901

[Nakagawa 1978] Y. Nakagawa, "On the Alexander polynomials of slice links", Osaka J. Math. 15:1 (1978), 161-182. MR 58 \#7600 Zbl 0401.57014

[Powell 2012] M. Powell, "A second order algebraic knot concordance group", Algebr. Geom. Topol. 12:2 (2012), 685-751. MR 2914616 Zbl 1244.57023

[Shaneson 1969] J. L. Shaneson, "Wall's surgery obstruction groups for $G \times Z$ ”, Ann. of Math. (2) 90 (1969), 296-334. MR 39 \#7614 Zbl 0182.57303

[Stallings 1965] J. Stallings, "Homology and central series of groups", J. Algebra 2 (1965), 170-181. MR 31 \#232 Zbl 0135.05201

[Vogel 1978] P. Vogel, "Localization of spaces with respect to a class of maps", preprint, Université de Nantes, 1978, Available at http://www.maths.ed.ac.uk/ aar/papers/vogelnantes.pdf.

[Wall 1999] C. T. C. Wall, Surgery on compact manifolds, 2nd ed., edited by A. A. Ranicki, Math. Surveys Monogr. 69, Amer. Math. Soc., Providence, RI, 1999. MR 2000a:57089 Zbl 0935.57003

Received October 28, 2013. Revised December 6, 2013.

JAE CHOON CHA

DePaRTMENT OF MATHEMATICS

PoHANG University of SCIENCE AND TECHNOLOGY

GYUNGBUK

POHANG 790-784

SOUTH KOREA

jccha@postech.ac.kr

and

SCHOOL OF MATHEMATICS

Korea Institute FOR AdVANCEd StUdy

SEOUL 130-722

SOUTH KorEA

MARK POWELL

DEPARTMENT OF MATHEMATICS

INDIANA UNIVERSITY

RAWLES HALL

831 EAST 3RD STREET

BLOOMINGTON, IN 47405

UNITED STATES

macp@indiana.edu 


\title{
PACIFIC JOURNAL OF MATHEMATICS
}

\author{
msp.org/pjm
}

Founded in 1951 by E. F. Beckenbach (1906-1982) and F. Wolf (1904-1989)

\section{EDITORS}

Don Blasius (Managing Editor)

Department of Mathematics

University of California

Los Angeles, CA 90095-1555

blasius@math.ucla.edu

\author{
Paul Balmer \\ Department of Mathematics \\ University of California \\ Los Angeles, CA 90095-1555 \\ balmer@math.ucla.edu \\ Robert Finn \\ Department of Mathematics \\ Stanford University \\ Stanford, CA 94305-2125 \\ finn@math.stanford.edu \\ Sorin Popa \\ Department of Mathematics \\ University of California \\ Los Angeles, CA 90095-1555 \\ popa@math.ucla.edu
}

\author{
Vyjayanthi Chari \\ Department of Mathematics \\ University of California \\ Riverside, CA 92521-0135 \\ chari@math.ucr.edu \\ Kefeng Liu \\ Department of Mathematics \\ University of California \\ Los Angeles, CA 90095-1555 \\ liu@math.ucla.edu \\ Jie Qing \\ Department of Mathematics \\ University of California \\ Santa Cruz, CA 95064 \\ qing@ cats.ucsc.edu
}

\section{PRODUCTION}

Silvio Levy, Scientific Editor, production@msp.org

\section{SUPPORTING INSTITUTIONS}

ACADEMIA SINICA, TAIPEI

CALIFORNIA INST. OF TECHNOLOGY

INST. DE MATEMÁTICA PURA E APLICADA

KEIO UNIVERSITY

MATH. SCIENCES RESEARCH INSTITUTE

NEW MEXICO STATE UNIV.

OREGON STATE UNIV.

\author{
STANFORD UNIVERSITY \\ UNIV. OF BRITISH COLUMBIA \\ UNIV. OF CALIFORNIA, BERKELEY \\ UNIV. OF CALIFORNIA, DAVIS \\ UNIV. OF CALIFORNIA, LOS ANGELES \\ UNIV. OF CALIFORNIA, RIVERSIDE \\ UNIV. OF CALIFORNIA, SAN DIEGO \\ UNIV. OF CALIF., SANTA BARBARA
}

\author{
Daryl Cooper \\ Department of Mathematics \\ University of California \\ Santa Barbara, CA 93106-3080 \\ cooper@math.ucsb.edu \\ Jiang-Hua Lu \\ Department of Mathematics \\ The University of Hong Kong \\ Pokfulam Rd., Hong Kong \\ jhlu@maths.hku.hk \\ Paul Yang \\ Department of Mathematics \\ Princeton University \\ Princeton NJ 08544-1000 \\ yang@math.princeton.edu
}

These supporting institutions contribute to the cost of publication of this Journal, but they are not owners or publishers and have no responsibility for its contents or policies.

See inside back cover or msp.org/pjm for submission instructions.

The subscription price for 2014 is US $\$ 410 /$ year for the electronic version, and \$535/year for print and electronic.

Subscriptions, requests for back issues and changes of subscribers address should be sent to Pacific Journal of Mathematics, P.O. Box 4163, Berkeley, CA 94704-0163, U.S.A. The Pacific Journal of Mathematics is indexed by Mathematical Reviews, Zentralblatt MATH, PASCAL CNRS Index, Referativnyi Zhurnal, Current Mathematical Publications and Web of Knowledge (Science Citation Index).

The Pacific Journal of Mathematics (ISSN 0030-8730) at the University of California, c/o Department of Mathematics, 798 Evans Hall \#3840, Berkeley, CA 94720-3840, is published twelve times a year. Periodical rate postage paid at Berkeley, CA 94704, and additional mailing offices. POSTMASTER: send address changes to Pacific Journal of Mathematics, P.O. Box 4163, Berkeley, CA 94704-0163.

PJM peer review and production are managed by EditFLOW ${ }^{\circledR}$ from Mathematical Sciences Publishers.

\section{PUBLISHED BY}

\section{mathematical sciences publishers \\ nonprofit scientific publishing}

http://msp.org/

(C) 2014 Mathematical Sciences Publishers 


\section{PACIFIC JOURNAL OF MATHEMATICS}

Volume $272 \quad$ No. $1 \quad$ November 2014

Nonconcordant links with homology cobordant zero-framed surgery $\quad 1$ manifolds

JAE CHOON CHA and MARK POWELL

Certain self-homotopy equivalences on wedge products of Moore spaces

Ho Won CHOI and KeE Young LeE

Modular transformations involving the Mordell integral in Ramanujan's lost notebook

Youn-SEO CHOI

The $D$-topology for diffeological spaces

J. DANiEl Christensen, Gordon SinNAMON and ENXIN Wu

On the Atkin polynomials

AHMAD EL-Guindy and Mourad E. H. ISMAIL

Evolving convex curves to constant-width ones by a perimeter-preserving flow

LAIYUAN GaO and Shengliang PAN

Hilbert series of certain jet schemes of determinantal varieties

SudhiR R. GHORPADE, Boyan Jonov and B. A. SeTHuraman

On a Liu-Yau type inequality for surfaces

Oussama Hijazi, Sebastián Montiel and Simon Raulot

Nonlinear Euler sums

ISTVÁN MEZŐ

Boundary limits for fractional Poisson $a$-extensions of $L^{p}$ boundary functions 227 in a cone

LEI QIAO and TAO ZHAO

Jacobi-Trudi determinants and characters of minimal affinizations

STEVEN V SAM

Normal families of holomorphic mappings into complex projective space concerning shared hyperplanes

LiU Yang, Caiyun Fang and Xuecheng Pang 